\title{
The Generation of Antiphase Oscillations and Synchrony by a Rebound-Based Vertebrate Central Pattern Generator
}

\author{
Wen-Chang Li, ${ }^{1}$ Robert Merrison-Hort, ${ }^{2}$ Hong-Yan Zhang, ${ }^{1}$ and Roman Borisyuk ${ }^{2,3}$ \\ ${ }^{1}$ School of Psychology and Neuroscience, University of St. Andrews, St Andrews, Fife, KY16 9TS, United Kingdom, ${ }^{2}$ School of Computing and Mathematics, \\ Plymouth University, Plymouth, Devon, PL4 8AA, United Kingdom, and ${ }^{3}$ Institute of Mathematical Problems in Biology, Russian Academy of Sciences, \\ 142290, Pushchino, Russia
}

\begin{abstract}
Many neural circuits are capable of generating multiple stereotyped outputs after different sensory inputs or neuromodulation. We have previously identified the central pattern generator (CPG) for Xenopus tadpole swimming that involves antiphase oscillations of activity between the left and right sides. Here we analyze the cellular basis for spontaneous left-right motor synchrony characterized by simultaneous bursting on both sides at twice the swimming frequency. Spontaneous synchrony bouts are rare in most tadpoles, and they instantly emerge from and switch back to swimming, most frequently within the first second after skin stimulation. Analyses show that only neurons that are active during swimming fire action potentials in synchrony, suggesting both output patterns derive from the same neural circuit. The firing of excitatory descending interneurons (dINs) leads that of other types of neurons in synchrony as it does in swimming. During synchrony, the time window between phasic excitation and inhibition is $7.9 \pm 1 \mathrm{~ms}$, shorter than that in swimming $(41 \pm 2.3 \mathrm{~ms})$. The occasional, extra midcycle firing of dINs during swimming may initiate synchrony, and mismatches of timing in the left and right activity can switch synchrony back to swimming. Computer modeling supports these findings by showing that the same neural network, in which reciprocal inhibition mediates rebound firing, can generate both swimming and synchrony without circuit reconfiguration. Modeling also shows that lengthening the time window between phasic excitation and inhibition by increasing dIN synaptic/conduction delay can improve the stability of synchrony.
\end{abstract}

Key words: central pattern generator; locomotion; oscillations; spinal cord; swimming; synchrony

\section{Introduction}

Multiple stereotyped behavioral patterns are common in both invertebrates and vertebrates. The underlying neural mechanisms have been better understood in some invertebrate preparations (Kupfermann and Weiss, 2001; Jing and Weiss, 2005; Kristan et al., 2005; Marder et al., 2005; Marder and Bucher, 2007; Briggman and Kristan, 2008). Studies show some neurons can be active in multiple tasks (Weimann et al., 1991; Weimann and Marder, 1994) and circuits can be dynamically reconfigured to generate different outputs (Dickinson et al., 1990; Meyrand et al.,

Received Oct. 1, 2013; revised Feb. 19, 2014; accepted March 21, 2014.

Author contributions: W.-C.L. designed research; W.-C.L., R.M.-H., and R.B. performed research; W.-C.L. and R.M.-H. analyzed data; W.-C.L., R.M.-H., H.-Y.Z., and R.B. wrote the paper.

This work was supported by the Wellcome Trust and a Royal Society Fellowship to W.-C.L. Many analyses on synchrony in this study took advantage of the big dataset accumulated in our past collaborations with Drs. Alan Roberts and Steve Soffe. This is essential since spontaneous synchrony is not common in normal conditions. The computational model was partially developed with support from a grant funded by UK Biotechnology and Biological Sciences Research Council. We thank Drs. Ying Zhang, Avis Cohen, Andrew McClellan, Keith Sillar, Alan Roberts, Steve Soffe, and David Parker for helpful discussions.

The authors declare no competing financial interests.

This article is freely available online through the J Neurosci Author Open Choice option.

Correspondence should be addressed to Wen-Chang Li at the above address. E-mail:wl21@st-andrews.ac.uk.

H.-Y. Zhang's present address: Centre for Neuroregeneration, University of Edinburgh, Edinburgh, UK.

DOI:10.1523/JNEUROSCI.4198-13.2014

Copyright (c) 2014 Li et al.

This is an Open Access article distributed under the terms of the Creative Commons Attribution License (http://creativecommons.org/licenses/by/3.0), which permits unrestricted use, distribution and reproduction in any medium provided that the original work is properly attributed.
1991, 1994; Popescu and Frost, 2002; Norris et al., 2006; White and Nusbaum, 2011). Much less is known about circuit architecture in vertebrates. It is widely thought that polymorphic neural networks can mediate multiple motor outputs (Getting, 1989; Soffe, 1993; Marder and Calabrese, 1996; Marder et al., 2005; Briggman and Kristan, 2008; Doi and Ramirez, 2008; Rauscent et al., 2009; Klein et al., 2010). However, a number of recent nonmammalian vertebrate studies suggest that circuit reconfiguration is needed to generate different motor responses (Ritter et al., 2001; Kimura et al., 2006; Berkowitz, 2007, 2008; Li et al., 2007; McLean et al., 2007, 2008; Liao and Fetcho, 2008; Frigon, 2009; Satou et al., 2009; Wyart et al., 2009; Berkowitz et al., 2010).

There are three basic categories of neural activity in central pattern generator $(\mathrm{CPG})$ circuits that control motor outputs: tonic firing, antiphase oscillations, and synchronous oscillations (termed "synchrony" here for simplicity). Movements like walking, running, and axial swimming involve antiphase oscillatory left-right and flexor-extensor muscle activities, known to be coordinated by reciprocal inhibition (Kiehn, 2006). In galloping, hopping, flying, frog- or turtle-style limb-based swimming, leftright synchrony is the operational mode. Whereas neural mechanisms underlying normal left-right synchrony have received little attention, synchrony has been reported in many experimental conditions (Cohen and Harris-Warrick, 1984; Clarke et al., 1991; Cowley and Schmidt, 1995; Kullander et al., 2003). In these studies, genetic mutations or pharmacological blockade led to 
functional reorganization of the circuits, and crossed excitation was proposed to couple left-right motor rhythms in synchrony.

Xenopus tadpoles have been used as a simple vertebrate model for studying neural mechanisms controlling locomotion, and the configuration of the tadpole spinal circuit has been systematically characterized recently using paired whole-cell recordings (Roberts et al., 2010). During tadpole swimming, neuronal activity alternates between the two sides of the spinal cord and hindbrain by way of reciprocal inhibition (Moult et al., 2013). Occasionally, however, the activity on the two sides can become spontaneously locked in synchrony, similar to that reported by Kahn and Roberts (1982) in curarized tadpoles. Synchrony disrupts propulsive swimming and bears no identified behavioral significance to tadpoles. We want to understand how synchrony rhythms are generated by this well studied neural circuit. In contrast to the prevalent evidence that circuit reconfiguration is needed for generating different network outputs, we reveal that the same CPG can generate both swimming and synchrony and that both types of rhythmic activity are coupled by fast reciprocal inhibition.

\section{Materials and Methods}

Experimental procedures were similar to those described previously (Li and Moult, 2012), approved by the local Animal Welfare Ethics Committee, and complied with UK Home Office regulations. Pairs of adult Xenopus of either sex were given injection of Human Chorionic Gonadotropin to induce mating. Tadpoles at stage 37/38 (Nieuwkoop and Faber, 1956), whose sex cannot be determined at this stage, were briefly anesthetized with $0.1 \%$ MS222 (3-aminobenzoic acid ester; Sigma-Aldrich) to allow immobilization with $\alpha$-bungarotoxin (12.5 $\mu \mathrm{M}$; Tocris Cookson) after the dorsal fin was cut open. The animal then was pinned onto a sylgard stage for dissections to expose myotome blocks, spinal cord, and caudal hindbrain. Additional dissections were then made to remove ependymal cells lining the central canal to reveal neuronal somata. Saline contained (in mM) $115 \mathrm{NaCl}, 3 \mathrm{KCl}, 2 \mathrm{CaCl}_{2}, 2.4$ $\mathrm{NaHCO}_{3}, 1 \mathrm{MgCl}_{2}$, and $10 \mathrm{HEPES}$, and $\mathrm{pH}$ was adjusted to 7.4 with $5 \mathrm{M}$ $\mathrm{NaOH}$. Current-clamp or voltage-clamp whole-cell recordings were made from exposed somata under a Nikon E600FN microscope. Patch pipettes were filled with $0.1 \%$ neurobiotin (Vector Laboratories) in the intracellular solution (in mM: $100 \mathrm{~K}$-gluconate, $2 \mathrm{MgCl}_{2}, 10 \mathrm{EGTA}, 10$ HEPES, $3 \mathrm{Na}_{2} \mathrm{ATP}$, $0.5 \mathrm{NaGTP}$, pH adjusted to 7.3 with $\mathrm{KOH}$ ). Pipette DC resistances ranged from 10 to $20 \mathrm{M} \Omega$. Standard current-clamp or voltage-clamp recordings were made with the Axon-2B or Axon Multiclamp 700B amplifier (Molecular Devices). Junction potentials (14.7 $\mathrm{mV}$ ) were not corrected in voltage-clamp recordings. Data were digitized using either CED 1401 plus or Power 1401 mkII, sampled with Signal 5 (Cambridge Electronic Design). Fictive swimming (we use "swimming" in the text for an easier description) was initiated by applying single or repetitive $1 \mathrm{~ms}$ current pulses to tadpole skin on the tail or head. Motor nerve (m.n.) recordings from muscle clefts were made normally between the 5 th and 10th muscle segments caudal to the otic capsule by using glass suction electrodes. Whole-cell recordings were made from neurons in the hindbrain (caudal to the fifth rhombomere segment) and rostral spinal cord (rostral to the fifth myotome). They were in current-clamp mode for looking at neuronal activities and in voltage-clamp mode for the examination of synaptic currents. Most neurons were successfully filled with neurobiotin after recording and stained as described previously (Li and Moult, 2012). Neuronal anatomy was used for final classification of neurons. Microperfusion of strychnine was done by positioning a glass pipette $(\sim 10 \mu \mathrm{m}$ in diameter $)$ in the hindbrain area. The microperfusion pipette was connected to a $50 \mathrm{ml}$ syringe where a gentle pressure $(\sim 0.01$ bar $)$ was kept.

Dynamic clamping was configured in Signal 5 to inject artificial synaptic currents into the recorded excitatory descending interneurons (dINs) to evoke extra midcycle firing. The currents were defined by an $\alpha$ synapse model and triggered by action potentials in the recorded dIN

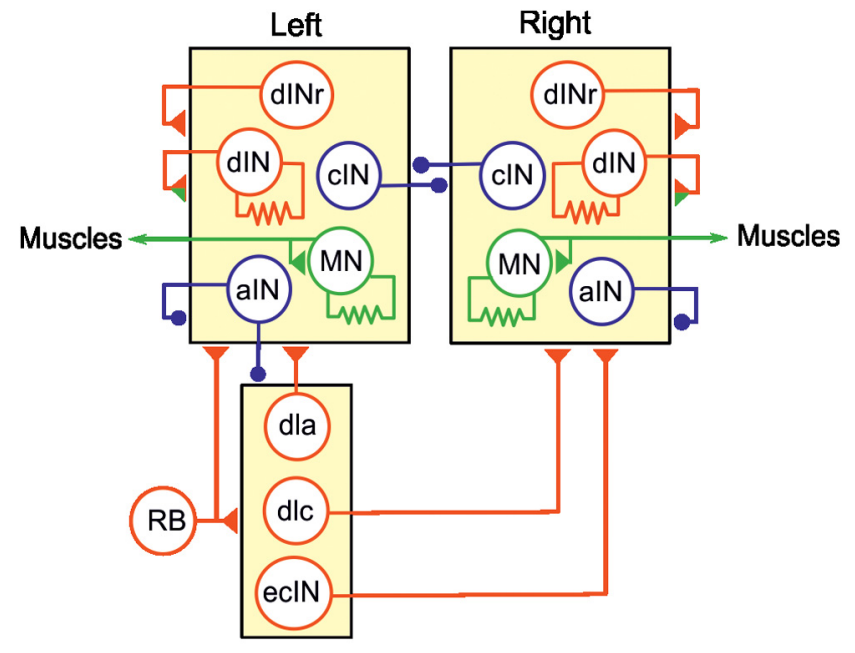

\section{- Glycine $\triangle A C h<$ Glutamate}

Figure 1. Tadpole spinal/hindbrain neurons and their synaptic connections. Rohon-Beard $(\mathrm{RB})$ neurons are the mechanosensory neurons that sense touch in the skin. Sensory interneurons include dlcs, dlas, and ecINs. Swimming CPG neurons include dINs, CINs, aINs, and MNs. dINrs and ecINs are only active during struggling (Li et al., 2007). The triangle synapse is excitatory, and the filled circle synapse is inhibitory. Synapses on the box means that all neurons inside the box receive inputs. Only sensory pathways on the left are shown to simplify the illustration.

(autapse) with a delay of 20-25 ms. Each dynamic-clamping period lasted about $200 \mathrm{~ms}$ to a few seconds.

Data processing and analyses were performed using Dataview (version 6.1, courtesy of Dr. W. J. Heitler, University of St. Andrews) and Excel. Synchrony activity was initially compared with swimming in recordings where a left dIN and a right m.n. were recorded simultaneously. In these recordings, dIN spike timing was adjusted to compensate for rostral-tocaudal delays and also dIN-to-m.n. activity delays (Roberts et al., 2010). dIN spike time was shifted for 6.5-11.2 ms so that dIN spikes appeared, on average, in the middle of swimming cycles defined by m.n. bursts (phase, 0.5 ). Neuronal firing reliability during swimming or synchrony is defined as the percentage of cycles with action potentials. The measurements of EPSCs and IPSCs were made by clamping dIN membrane potential at approximately -55 or $0 \mathrm{mV}$, respectively. The size of EPSCs and IPSCs during synchrony was compared with that in swimming immediately before or after the synchrony bouts. Statistical analyses were done using PASW statistics 18 (SPSS). Normality tests were performed first, and means were given with SEs (Mean \pm SEM) and compared using $t$ tests for normally distributed data. The Wilcoxon signed rank test was used for other datasets unless otherwise stated.

The tadpole swimming model represented a $1.5 \mathrm{~mm}$ section of tadpole spinal cord with $\sim 1500$ neurons, interconnected by $\sim 85,000$ synapses. Synaptic connectivity was generated by our anatomical model combined with a developmental model of neuron growth (Borisyuk et al., 2011, 2014; Roberts et al., 2014). The general organization of synaptic connectivity in the model is in line with the schematic representation shown in Fig. 1. Neuron dynamics were based on conduction-based HodgkinHuxley equations defined previously (Sautois et al., 2007), but with various adjustments as described previously by Roberts et al. (2014), namely (1) the addition of hyperpolarization-activated calcium channels (Dale, 1995), whose dynamics are given by the Goldman-Hodgkin-Katz equation; (2) adjusted ion channel gating dynamics that better match dIN firing properties such as a single action potential at the onset of fast depolarization, rebound firing after fast inhibition when depolarized, and repetitive firing when NMDARs are activated (Li et al., 2006, 2010); and (3) the addition of gap junctions with a conductance of $0.2 \mathrm{nS}$ between dINs within $100 \mu \mathrm{m}$ of each other in the rostrocaudal direction. Unlike the model dINs of Roberts et al. (2014), we did not include a fast potassium current but increase maximum slow potassium conductance from 9.6 to $150 \mathrm{~ns}$, as voltage-clamp recordings showed that potassium 
A

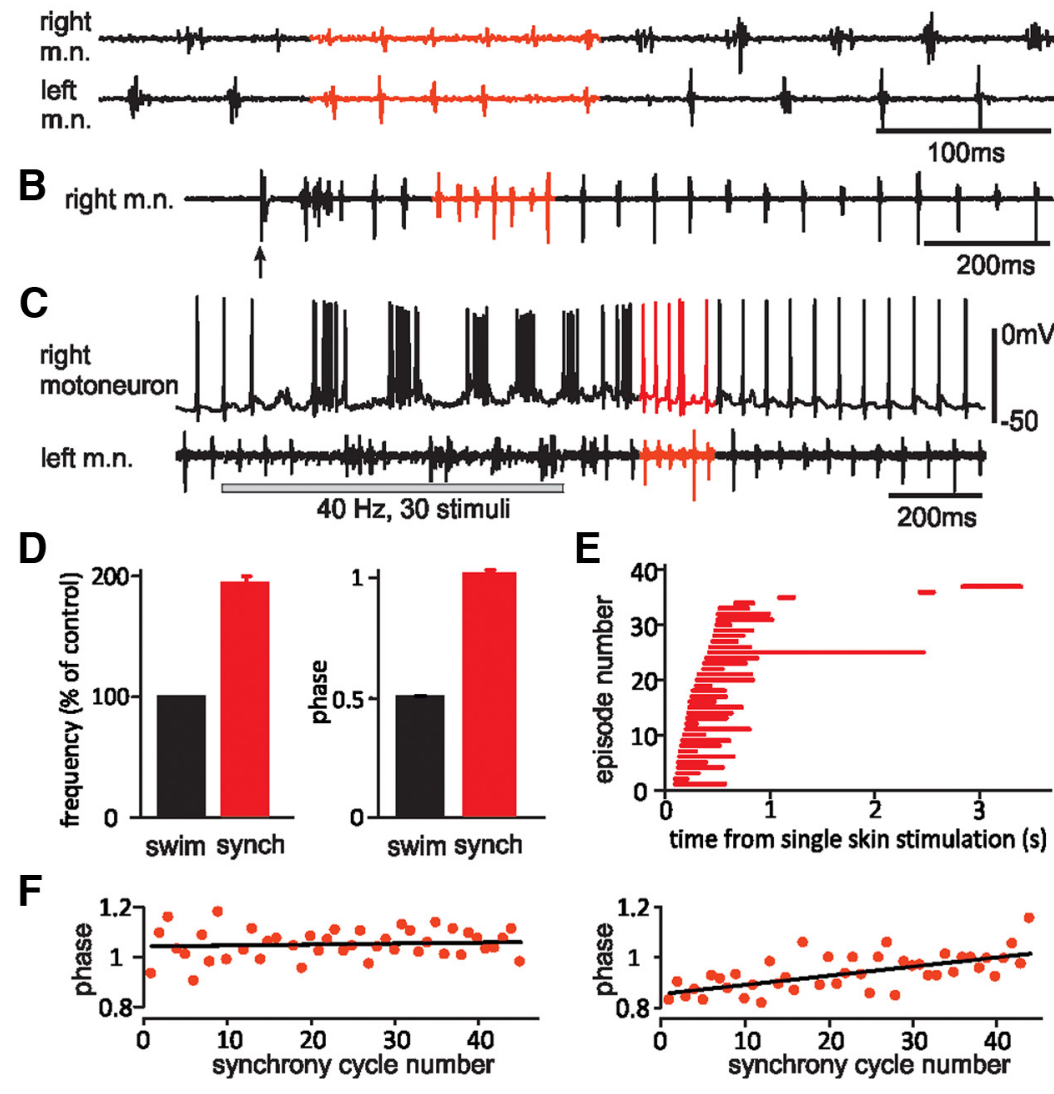

Figure 2. Left-right motor synchrony in tadpoles and its basic features. $A, A$ synchrony bout with left and right m.n. activity recorded simultaneously (both fifth/sixth muscle cleft). B, Synchrony after single skin stimulation (arrow). C, Synchrony after repetitive skin stimulation (gray bar) with a simultaneous motoneuron recording. $\boldsymbol{D}$, Synchrony rhythm frequency and phase (red bars) compared with that of adjacent swimming (black bars). $\boldsymbol{E}$, Starting time (beginning of red lines) and duration (length of lines) for 37 synchrony bouts after single skin stimulation in 13 tadpoles. $F$, Cycle-by-cycle phase measurements of two synchrony bouts. Left, Example lacking a phase drift (linear regression coefficient is 0.0004 per cycle; $R^{2}=0.006 ; p=0.62$ ). Right, Example with the clearest phase drift with the progression of synchrony (coefficient is 0.0036 per cycle; $R^{2}=0.41 ; p<0.001$ ). Solid lines are for linear regression. Synchrony bouts are red traces, and swimming activity is black in this and other figures.

sural interneurons (cINs) (from 0.593 to 0.65 $\mathrm{nS}$ ) and motoneurons (MNs) (from 0.593 to $0.54)$. For the same reason, we made a larger change to the strength of dIN to ascending interneuron (aIN) synapses (from 0.1 to $0.3 \mathrm{nS}$ ). Finally, the conductance of the inhibitory synapses made by aINs onto all other neuron types was reduced to $0.0435 \mathrm{nS}$ so that these synapses provided only $10 \%$ of the inhibitory strength of corresponding cIN synapses.

As in the study by Roberts et al. (2014), all synaptic connections contained a constant delay of $1 \mathrm{~ms}$ and a conduction delay that depends on the difference in rostrocaudal position of the presynaptic and postsynaptic neurons according to a conduction speed of 3.5 $\mathrm{ms} / \mathrm{mm}$.

The simulation was implemented using custom $\mathrm{C}$ code. The simulator uses the fourthorder adaptive Runge-Kutta-Felberg ODE solver from the GNU Scientific Library (version 1.15). The absolute and relative error tolerances for step-size control and adjustment were $1.0 e^{-5}$, and spikes are detected by membrane potential zero crossing after every time step (maximum step size, $0.5 \mathrm{~ms}$ ). Small variability in neuron and synapse strengths was introduced by adding Gaussian noise (SD of $2 \%$ of mean values) to cell membrane capacitance and channel conductances and Gaussian noise (SD of $5 \%$ of mean values) to the conductance of each synapse.

\section{Results}

Basic features of synchrony in tadpoles Left-right synchrony in tadpoles involves simultaneous m.n. bursts on both sides, in contrast to the alternation during swimming (Fig. 2A). We first analyzed the basic properties of synchrony. We arbitrarily define periods with more than five consecutive bursts of synchronous activity

currents in dINs lack a fast component (W.-C. Li, unpublished observations). All other parameters associated with neurons' membrane properties were the same as given by Roberts et al. (2014).

The model includes glutamatergic (AMPARs, NMDARs with $\mathrm{Mg}^{2+}$. dependent voltage dependency) and glycinergic synapses established in paired whole-cell recordings (Sautois et al., 2007). We introduced synaptic saturation in the present model by increasing synaptic opening $o(t)$ and closing $c(t)$ variables using a varying, rather than constant, increment after a spike. The postspike increment $\Delta_{o, c}(t)$ is given by the following equation:

$$
\Delta_{o, c}(t)=s\left(1-\frac{c(t)-o(t)}{\sigma}\right)
$$

Here parameter $s$ is the original increment size (AMPA/NMDA, 1.25; glycine, 3.0), and $\sigma$ is the saturation level (AMPARs and glycine receptors, 1.0; NMDA, 5.0). These values were chosen so that saturation only had a major effect on NMDAR activation during high-frequency activity.

In most cases, we used the same synaptic parameters as given by Roberts et al. (2014), but in some cases, minor changes were made. First, to obtain realistic and stable swimming activity after removal of the fast potassium current in dINs and to enable synaptic saturation, it was necessary to increase the strength of dIN-dIN NMDA synapses from 0.15 to $0.29 \mathrm{nS}$. This new value agrees with the standard NMDA conductance given by Sautois et al. (2007). To obtain realistic measures of firing reliability of CPG neurons during swimming and synchrony, we made small changes to the conductances of synapses that dINs make onto commis- (four cycles) as a synchrony bout. Initially, we analyzed spontaneous synchrony bouts in 14 tadpoles where the activity of a left m.n. (normally eighth/ninth myotome cleft) and a right dIN in the caudal hindbrain was recorded simultaneously (see Materials and Methods; $n=17$ synchrony bouts, 419 cycles). Periods of swimming were chosen either immediately before or after the synchrony for comparison (175 cycles). During synchrony, the phase of left-side activity relative to the right side was 1 (1.01 \pm 0.01 ; one-sample, two-tailed $t$ test, $p=0.18$; Fig. $2 A, D$ ), indicating activity on the two sides was synchronous. In 13 long bouts with $30 \pm 4$ cycles (range, 14-62), we examined whether one side clearly led the synchronous activity. In four bouts, there were $>80 \%$ of cycles with phase value above or below 1 , indicating one side being active consistently earlier than the other side (Fig. 2F, left). If synchrony maintenance relies on the synchronous firing of neurons in the network, the gradual drifting of phase during synchrony may end synchrony itself. Linear regression was applied to the phase measurements of each of the 13 long bouts to see whether there was drifting of phase with the progression of synchrony. Significance was found in 6 of the 13 bouts. The drifting rate (regression coefficient) was $0.0029 \pm 0.0008$ per cycle, equivalent to $0.11 \pm 0.03 \mathrm{~ms}$ assuming one synchrony cycle is about $40 \mathrm{~ms}$. In three bouts, the leading side remained the same but with increased phase lags, whereas in the other three, it lost its 
Table 1. Neuronal firing reliability (percentage of cycles with firing) during synchrony and adjacent swimming

\begin{tabular}{llll}
\hline & & \multicolumn{2}{l}{$\begin{array}{l}\text { Firing reliability (\%), mean } \pm \text { SEM } \\
\text { or mean (median) }\end{array}$} \\
\cline { 3 - 4 } Cell type & $\begin{array}{l}\text { Number } \\
\text { of cells }\end{array}$ & Synchrony & Swimming \\
\hline Swimming CPG & & & \\
dIN & 19 & $84(100)^{*}$ & $99(100)$ \\
Motoneuron & 13 & $65 \pm 7.3^{*}$ & $86(100)$ \\
aIN & 13 & $45 \pm 9^{* *}$ & $64 \pm 9$ \\
CIN & 10 & $52 \pm 8^{* * *}$ & $73 \pm 9$ \\
Other types & & & \\
dlc & 8 & 0 & 0 \\
dla & 10 & 0 & 0 \\
dINr & 11 & 0 & 0 \\
eclN & 6 & $5(0)$ & $2(0)$ \\
\hline
\end{tabular}

The identification of dINrs and ecINs was based on their synaptic outputs in paired recordings, activity during struggling, and anatomy (Li et al., 2007). Significance levels are as follows: ${ }^{*} p<0.05 ;{ }^{* *} p<0.01 ;{ }^{* * * *} p<0.001$.

lead toward the end of synchrony (Fig. $2 F$, right). The small phase drifts $(0.07 \pm 0.02 ; n=6$, estimated by regression $)$ all ended abruptly with phases jumping to $\sim 0.5$ when synchrony switched to swimming. Synchrony frequency $(24 \pm 0.6 \mathrm{~Hz})$ was reliably twice the frequency of adjacent swimming $(12.4 \pm 0.4 \mathrm{~Hz}$; $195.5 \pm 4.5 \%$; one-sample, two-tailed $t$ test, $p=0.34$; Fig. $2 D)$. This feature of abruptly-doubled m.n. frequency fits in with previous descriptions (Clarke et al., 1991). Since swimming frequency only changes gradually after initiation, we used this simple unique feature to identify synchrony when activity was not recorded simultaneously from both sides.

In a bigger sample of 37 tadpoles, we analyzed how often and when synchrony occurred. Within $6 \mathrm{~s}$ after single skin stimulation, 37 synchrony bouts were seen in 13 tadpoles $(17.5 \%$ of 212 trials; Fig. $2 B$ ). Within $2.5 \mathrm{~s}$ after repetitive skin stimulation (30-40 Hz, 20-50 pulses), 50 bouts of synchrony were seen in 22 tadpoles (26.6\% of 188 trials; Fig. 2C). Overall, we observed synchrony in 26 of 37 tadpoles (70.3\%) when both types of stimulation were considered. The 37 synchrony bouts evoked by single skin stimulation lasted $0.35 \pm 0.03 \mathrm{~s}$ (range, $0.09-2.04$ ) or $11 \pm$ 0.8 cycles (range, $4-66$ ). Most of them started within $0.5 \mathrm{~s}$ and stopped within $1 \mathrm{~s}$ after skin stimulation (32 of 37; Fig. $2 E$ ). When the hindbrain was sectioned at the fifth/sixth rhombomere border in another sample of 11 tadpoles, synchrony was seen in 1 of 82 trials after single skin stimulation and in 59 of 521 trials after repetitive skin stimulation of nine tadpoles. This suggests that tadpole spinal cord and caudal hindbrain, which contain the highest density of dINs and are critical for swimming (Li et al., 2006; Soffe et al., 2009), can also generate synchrony.

\section{Neuronal activity during synchrony}

To understand how synchrony is generated, we next identified neurons that were active during synchrony. We found that only neurons that were rhythmically active during swimming fired action potentials during synchrony (Table 1). They included all swimming CPG neurons (Roberts et al., 2010): dINs, cINs, aINs, and motoneurons (Fig. 3). We define their firing reliability as the percentage of cycles with action potentials. Their firing reliabilities during synchrony were lower than those during swimming immediately before or after synchrony (Table 1). To see whether there is a change of firing reliability with the progression of synchrony, we compared the reliability in the first five synchrony cycles with that in the last five cycles. In 26 long bouts with an average of $19.4 \pm 1.3$ synchrony cycles (range, 12-35) from 11 neurons ( 3 motoneurons, 4 dINs, 2 cINs, and 2 aINs), no differ- ence was found (paired $t$ test, $p=0.74$ ). Neurons that were silent during swimming [dorsolateral commissural interneurons (dlcs), dorsolateral ascending interneurons (dlas), excitatory commissural interneurons (ecINs), and repetitive-firing descending interneurons (dINrs)] remained silent during synchrony (Table 1). This lack of recruitment or exclusion of network neurons between the two motor patterns strongly suggests that synchrony is a motor output derived from the swimming circuit.

\section{Analyses of dIN activity during synchrony}

dINs are the only excitatory interneurons in the swimming CPG, and their activity drives swimming (Soffe et al., 2009; Li, 2011). We made 11 paired whole-cell recordings with one dIN and another (non-dIN) CPG neuron at similar locations on the same side. This allowed us to determine directly which neuron fired first in each synchrony or swimming cycle. dIN firing during synchrony preceded the firing of non-dINs (seven cINs, two motoneurons, two unidentified). The latency between dIN and non$\mathrm{dIN}$ firing in synchrony ( $2.6 \pm 0.68 \mathrm{~ms} ; n=11$ bouts) was similar to that in swimming $(2.47 \pm 0.46 \mathrm{~ms} ; p>0.05, t$ test; Fig. $4 A-C)$. Since there was no recruitment of extra types of neurons in synchrony (see above), this suggests that the excitatory dINs were also responsible for driving the firing of other neurons during synchrony, as they are for swimming (Soffe et al., 2009). dIN rebound firing had been proposed as the main mechanism in sustaining swimming (Moult et al., 2013). To see whether the same rebound mechanism could sustain synchrony, we measured the delay between the start of IPSPs and the time point when the following dIN spike crossed $0 \mathrm{mV}$. This time (rebound time) should reflect the interaction between different voltagedependent ionic channels and receptor channels that give rise to dIN rebound firing. No difference was found between rebound time during swimming $(36.3 \pm 2.6 \mathrm{~ms})$ and that during synchrony $(34.3 \pm 2.2 \mathrm{~ms} ; n=7 \mathrm{dINs}$; two-tailed paired $t$ test, $p>0.05$; Fig. $4 D, E)$. These data suggest that the same rebound mechanism that underlies swimming is also responsible for synchrony.

\section{Synaptic currents in dINs during synchrony}

Knowing that dIN activity is likely to drive synchrony, we next looked at the synaptic currents dINs receive during synchrony to understand how they could support dIN firing. dINs receive excitation from other dINs during swimming, with NMDAR components contributing to tonic inward currents and AMPAR and nicotinic receptor components giving rise to the phasic on-cycle EPSCs (Fig. 5A). Additionally, cINs from the opposite side produce midcycle IPSCs, and ipsilateral aINs produce early-cycle IPSCs, which appear between the on-cycle dIN EPSCs and cIN IPSCs (Li and Moult, 2012; Fig. 5B, arrows). First, membrane potential was clamped around IPSC reversal (approximately -55 $\mathrm{mV}$ ) to compare EPSCs. EPSCs were smaller in synchrony $(-119 \pm 13 \mathrm{pA})$ than those in swimming just before or after synchrony $(-150 \pm 19 \mathrm{pA} ; n=11$ bouts in 11 neurons; paired $t$ test, $p<0.01$; Fig. 5A). Second, membrane potentials were clamped around $0 \mathrm{mV}$ to reveal IPSCs. During synchrony, aINs and cINs fire synchronously, and both can contribute to the IPSCs. The mixed IPSCs during synchrony (262 $\pm 42 \mathrm{pA})$ were smaller than the midcycle cIN-mediated IPSCs in swimming ( $322 \pm 51 \mathrm{pA} ; n=8$ bouts; paired $t$ test, $p<0.05$; Fig. $5 B)$. This fits with the observation above that neuronal firing during synchrony is less reliable than during swimming (Table 1). Third, the delay between EPSCs and IPSCs in swimming or synchrony cycles was measured when membrane potential was clamped at 
A
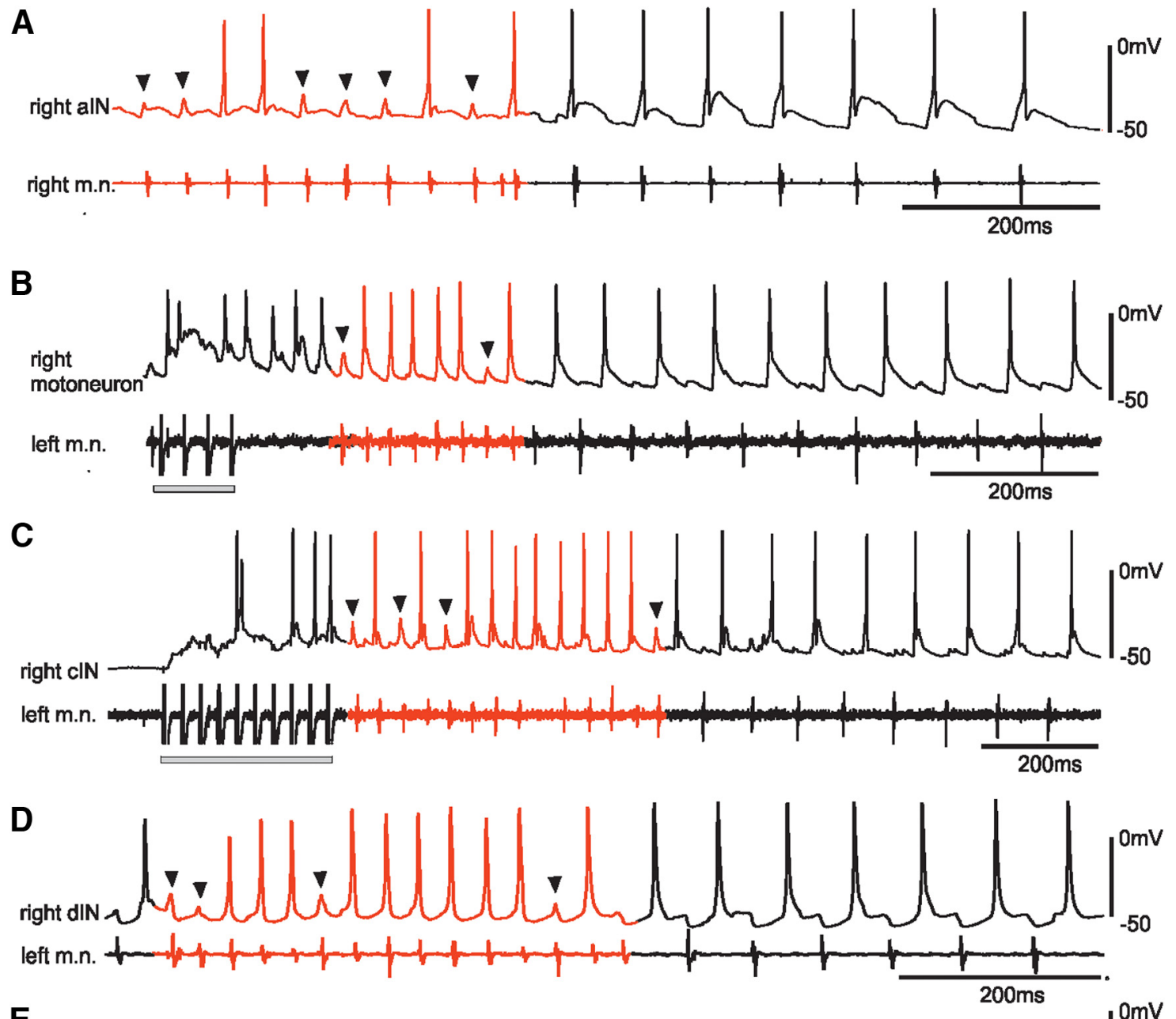

E

right dlc

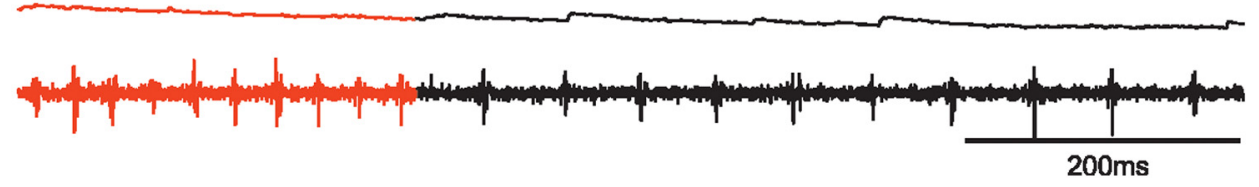

Figure 3. Activity of spinal and hindbrain neurons during synchrony and swimming. Four types of $C P G$ neurons $(\boldsymbol{A}$, alN; $\boldsymbol{B}$, motoneuron; $\boldsymbol{C}, \mathrm{cIN} ; \boldsymbol{D}, \mathrm{dIN})$ and a dlc $(\boldsymbol{E})$ are illustrated. $\operatorname{Gray}$ bars indicate periods of repetitive skin stimulation $(30 \mathrm{~Hz})$. Note the abrupt doubling and halving of rhythm frequencies when synchrony starts and ends. Synchrony cycles with failure in spiking are marked with filled triangles.

approximately $-20 \mathrm{mV}$. This was measured as the time difference between the EPSC trough and the IPSC peak. The delay was $7.9 \pm 1$ ms during synchrony, which was much shorter than that during swimming ( $41 \pm 2.3 \mathrm{~ms} ; n=8$ bouts; paired $t$ test, $p<$ 0.01 ; Fig. $5 C$ ). This delay is important because if inhibition arrives during the rising phase of a dIN action potential, then the spike may be suppressed, which reduces spike reliability (Table 1; Figs. $3 D, 5 D)$. Instead, inhibition must arrive during the decaying phase of the dIN action potential to trigger another postinhibitory spike. The fact that during synchrony IPSCs arrive very shortly after dIN spiking may contribute to the instability of synchrony, since a small difference in the timing of inhibition may cause it to arrive before dIN spiking.

\section{Midcycle firing in dINs may initiate synchrony}

A critical feature of synchrony is that its frequency is double that of swimming. Abnormal midcycle dIN firing, which doubles the frequency of dIN activity, may initiate synchronous activity because dIN activity drives the network. We looked at the incidences of single, spontaneous midcycle firing (Fig. 6A) in seven paired recordings from a dIN and a non-dIN at similar locations on the same side (non-dINs include one motoneuron and six cINs). These recordings allowed us to determine whether dINs or non-dINs were more likely to fire midcycle spikes. One hundred and ten midcycle firing events were found (in $>5000$ cycles) without evidence for synchrony in the m.n. recordings. In 81 cases $(74 \%)$, the dIN fired midcycle action potentials without simultaneous midcycle firing in non-dINs. In 23 cases $(21 \%)$, both the dINs and non-dINs fired. Only non-dINs fired action potentials in the remaining six cases $(5 \%)$. The higher probability of dIN midcycle firing supports the proposal that occasional dIN spiking may initiate midcycle activity and synchrony. The phase of midcycle dIN spikes in the swimming cycle was $0.54 \pm 0.004$ $(n=104)$. This was not different from the phase for midcycle 

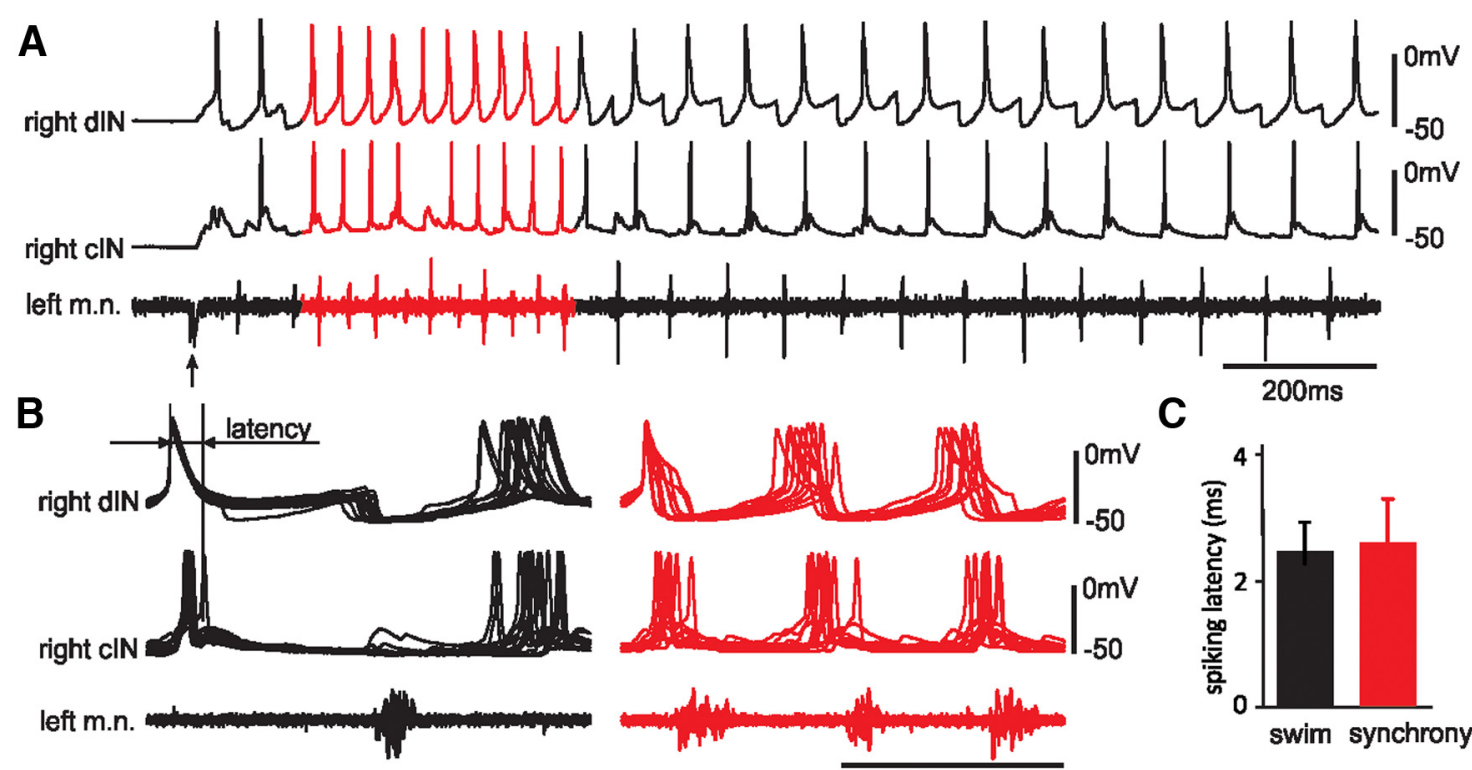

C
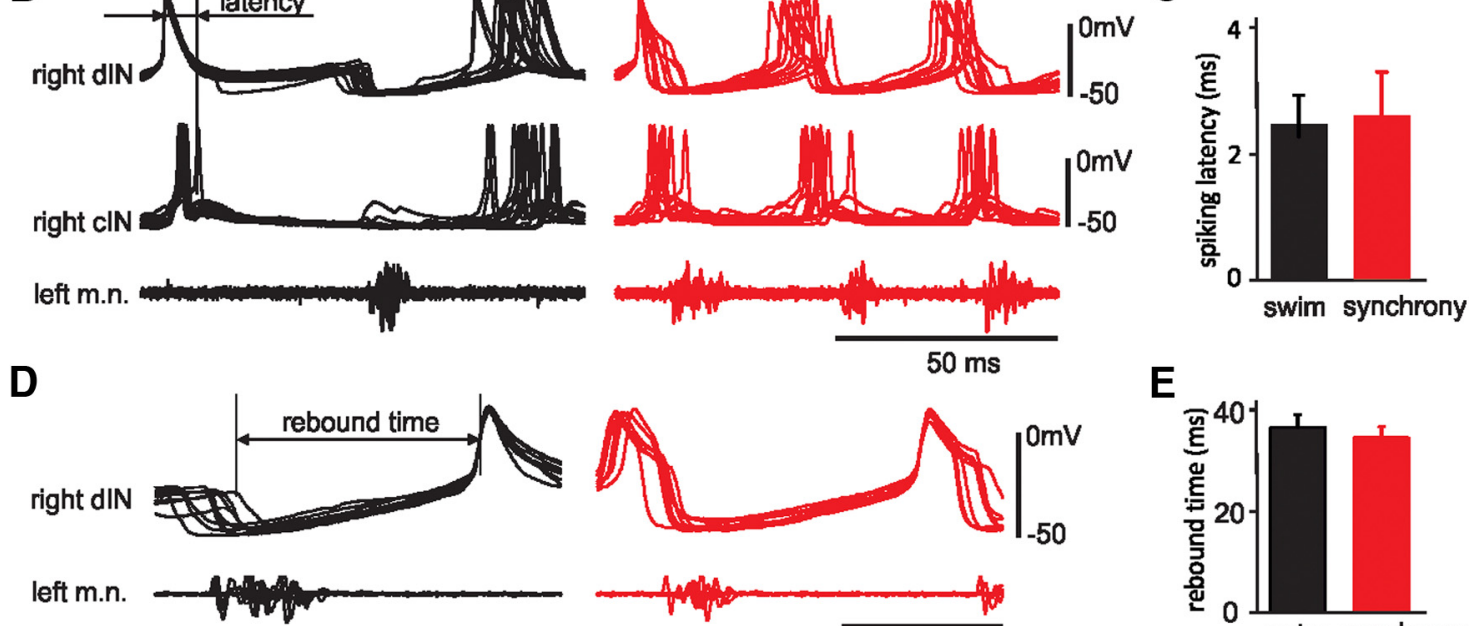

E
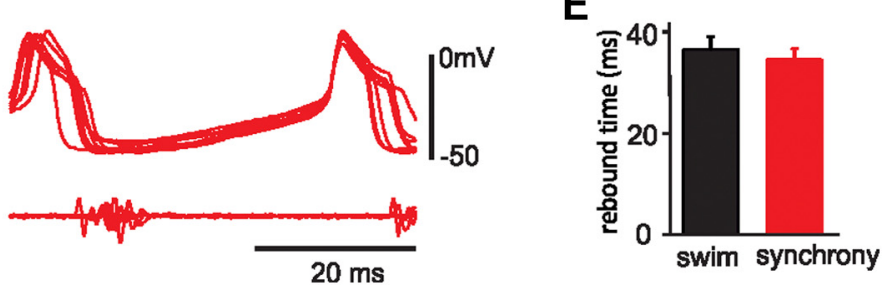

Figure 4. Analyses of dIN activity during swimming and synchrony. $\boldsymbol{A}$, A synchrony bout shortly after swimming is started by single-pulse skin stimulation (arrow) in a paired recording. $\boldsymbol{B}$, Overlapped traces of swimming and synchrony cycles from $A$ at a smaller time scale to show the time of neuronal firing. Traces are lined up to the rising phase of the first dIN spike. Note dIN spikes reliably precede cIN spikes in both swimming and synchrony cycles. Latency is the time difference when spikes cross $0 \mathrm{mV}$. C, Spiking latencies between dINs and other CPG neurons in paired recordings are not different during swimming and synchrony. $\boldsymbol{D}$, Rebound time measured from the start of IPSPs to the time when dIN spikes cross $0 \mathrm{mV}$. $\boldsymbol{E}$, Rebound time for swimming and synchrony is not different.

IPSPs in dINs (median, $0.54 ; n=308$ cycles in five tadpoles analyzed; $p=0.69$, independent sample nonparametric median test). However, a dIN spike can appear a few milliseconds earlier than IPSPs in individual cases (Fig. $6 \mathrm{~B}$ ), allowing it to drive postsynaptic firing. Therefore, this type of abrupt doubling of dIN firing frequency during swimming in the form of midcycle spikes may be key to synchrony initiation.

As we have shown above, most synchrony bouts occur within $1 \mathrm{~s}$ of skin stimulation. Synaptic currents during this initial period differ from the following periods in that there are bigger tonic inward currents and more reliable aIN IPSCs in dINs (Li and Moult, 2012). Higher depolarization can promote firing at midcycle position. We injected positive DC (60-200\% threshold current for evoking firing at rest) into $15 \mathrm{dINs}$ to mimic higher background excitation to see whether this could induce midcycle dIN firing during swimming. In three dINs, this led to doublet on-cycle firing in 48 of 49 swimming cycles. In the other $12 \mathrm{dINs}$, DC injection led to midcycle dIN spikes on many cycles (Fig. 6C; 269 of 796 cycles). In three trials in three different dINs, this DC injection coincided with synchrony during the injection period (Fig. 6F). However, such coincidence was not repeatable. Injecting DC into non-dINs (55-200\% threshold current for firing; five motoneurons, five cINs, four aINs, and two unidentified CPGs) mainly induced on-cycle multiple firing ( 265 of 502 cycles) with occasional midcycle firing (Fig. $6 D ; 31$ cycles). There were higher percentages of cycles with midcycle firing in dINs $(p<0.01)$ and multiple firing in non-dINs ( $p<0.001$, independent samples median test; Fig. $6 E$ ). These results indicate that dINs, most of which were recorded in the caudal hindbrain and rostral spinal cord, tend to fire midcycle spikes during enhanced excitation. In some cases, the midcycle firing of dINs may directly lead to synchrony.

The midcycle dIN firing evoked by DC current injections during swimming tended to appear in the latter half-cycle (Fig. $6 \mathrm{C}$, filled triangles), potentially reducing its ability to evoke synchrony. This asymmetrical firing pattern is presumably caused by different synaptic events in a swimming cycle, first strong reliable on-cycle dIN excitation and then weak unreliable early-cycle aIN inhibition and strong reliable midcycle cIN inhibition ( $\mathrm{Li}$ and Moult, 2012; Fig. 5B). To have better control over the timing of evoked midcycle dIN firing, we used the dynamic-clamp technique to time the injection of artificial excitatory synaptic currents into the recorded dINs. The synaptic currents were triggered by the dIN action potentials with an artificial delay of approximately half of the swimming cycle period $(20-25 \mathrm{~ms})$. We failed to induce any synchrony bouts in this way ( $n=12 \mathrm{dINs}, 259$ trials, $3-50$ cycles per trial with current injections, data not shown).

\section{Effects of blocking inhibition on synchrony generation}

Blocking glycinergic inhibition can induce synchrony in neonatal rats (Cowley and Schmidt, 1995) and lamprey (Cohen and Harris-Warrick, 1984). In tadpoles, bath-applied $2.5 \mu \mathrm{M}$ strychnine and $20 \mu \mathrm{M}$ SR95531 (GABA antagonist) led to an initial silent period during which initiation of swimming by single skin stimulation was very difficult. After $\sim 23 \mathrm{~min}$, the rhythms recovered to some extent after multiple skin stimulation, but with little synchrony (Moult et al., 2013). Application of lower concentra- 
A
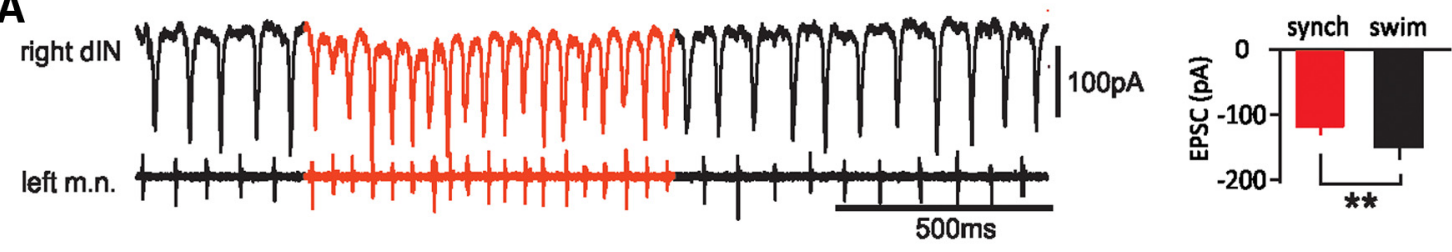

B
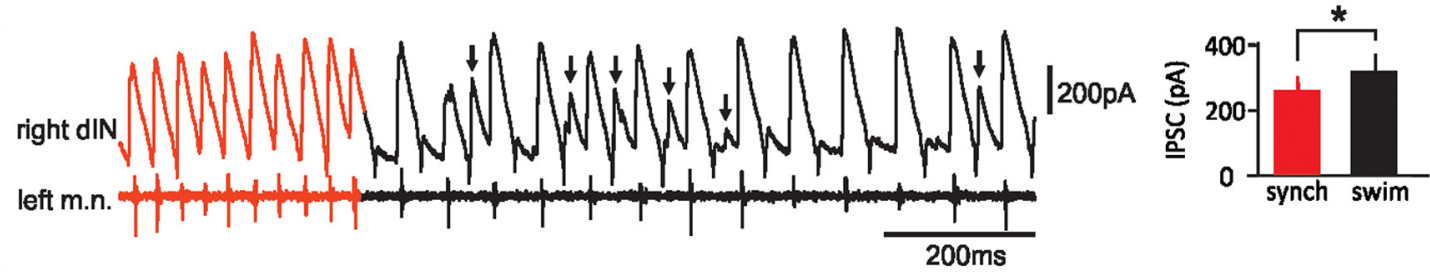

C
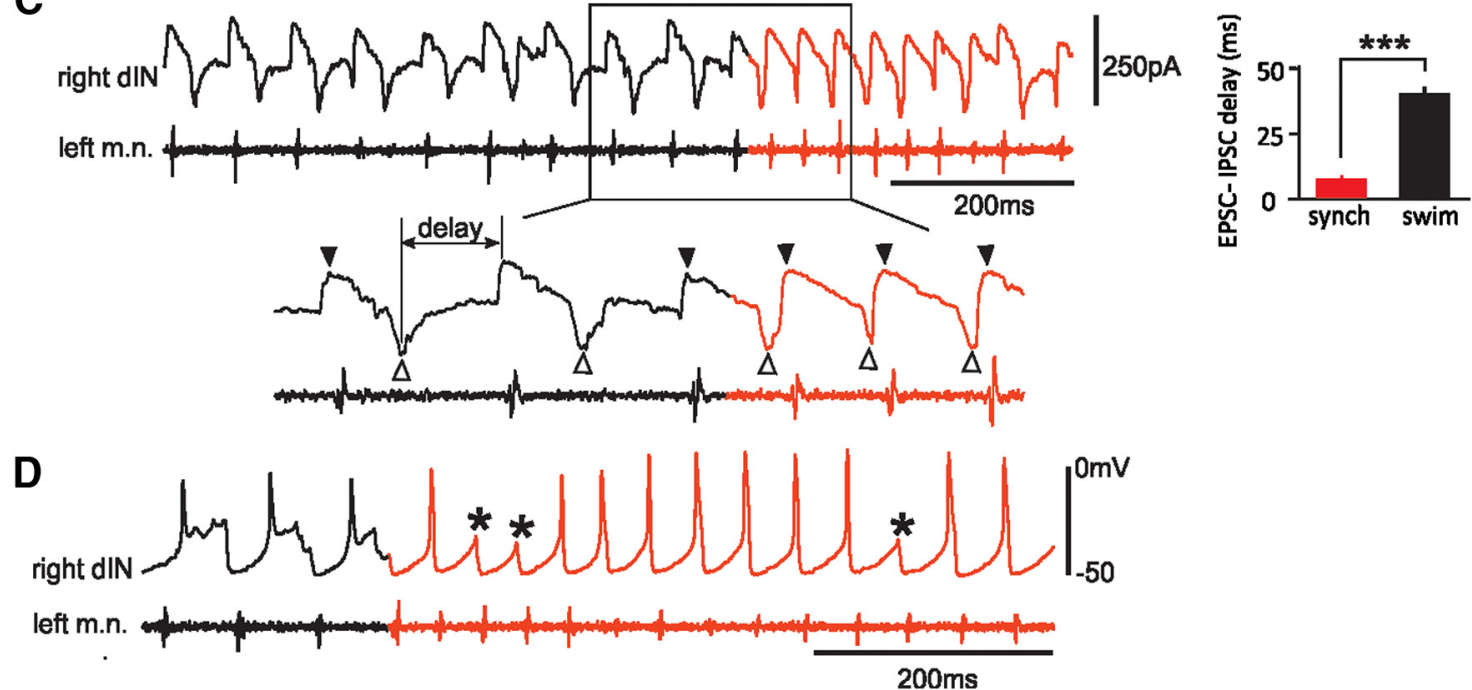

Figure 5. Synaptic currents and potentials in dINs during synchrony and swimming. $A$, Rhythmic on-cycle EPSCs (membrane potential clamped at approximately $-55 \mathrm{mV}$ ). The right bar chart summarizes averaged measurements in 11 neurons. $\boldsymbol{B}$, Rhythmic IPSCs (membrane potential clamped at $\sim 0 \mathrm{mV}$ ). The bar chart is for averaged midcycle IPSC measurements in eight neurons. Arrows point at early-cycle IPSCs from alNs. C, Delay from on-cycle EPSC peak (triangles) to midcycle IPSC peak (filled triangles). The right bar chart shows the delay is much shortened during synchrony compared with that in swimming. Significance values as follows: ${ }^{*} p<0.05 ;{ }^{* *} p<0.01 ;{ }^{* * *} p<0.001$. D, dIN spiking is inhibited by IPSPs on some cycles during a synchrony bout (asterisks). dINs are recorded in voltage-clamp mode $(\boldsymbol{A}-\boldsymbol{C})$ and in current-clamp mode $(\boldsymbol{D})$. Synch, Synchrony.

tions of strychnine $(0.07-0.25 \mu \mathrm{M})$ increased swimming frequency, but the incidence of synchrony did not increase ( $\mathrm{Li}$ and Moult, 2012).

Fortuitously, two batches of tadpoles showed an unusual tendency for synchronous activity (Fig. 7A; data were not included for preparing Fig. $2 E$ ). In these animals, $21.1 \pm 4.6 \%$ of rhythmic activity (including both synchrony and swimming) was synchrony ( $n=63$ swimming episodes). Microperfusing $0.5 \mu \mathrm{M}$ strychnine in the hindbrain area decreased synchrony to $1.8 \pm$ $0.6 \%$ ( $n=66$ episodes, 7 tadpoles; $p<0.01$, paired $t$ test $)$. This recovered to $23.3,17.8$, and $5.3 \%$ in wash in three tadpoles $(>20$ min; Fig. 7A). Injecting positive DC in two of the seven dINs, which could induce midcycle dIN firing in control (cycles with midcycle firing are 33 of 57 and 23 of 115 , respectively) as shown above, evoked little dIN midcycle firing in strychnine ( 0 of 20 and 1 of 84 ; Fig. $7 B$ ). These data imply that inhibition may play an important role in the generation of synchrony.

Synchrony initiation and stability in a computational model of swimming

The excitation from single midcycle spiking in one dIN in wholecell recordings above was very unlikely to be sufficient to drive substantial midcycle cIN firing and start synchrony. Therefore, we injected brief midcycle currents into multiple dINs simultaneously in a computational tadpole swimming network to see whether synchrony could be initiated.

We performed multiple simulations of the neuronal network model to investigate the dynamical characteristics of synchrony and swimming. Importantly, there was only reciprocal inhibitory connection between the left and right sides (Fig. 1). The network displays stable and realistic swimming activity that is initiated by stimulation of sensory neurons. We injected brief step currents ( 5 $\mathrm{ms}, 150 \mathrm{pA}$ ) into dINs on the right side during swimming once to make them fire midcycle spikes. Though injections into as few as 10 dINs (still too many to be able to manipulate experimentally here) could evoke brief synchrony bouts, the most stable synchrony was evoked when all dINs were injected with currents. The synchrony bouts evoked by current injections into all dINs lasted for an average of 16.1 cycles (median, 11 ; range, $6-47 ; n=$ 12 trials; Fig. 8A). The respective swimming and synchrony frequencies were very similar across trials and remained stable. Autocorrelation of motoneuron activities on one side across 12 trials gave an average swimming frequency of $20.4 \mathrm{~Hz}$ and a synchrony frequency of $43.7 \mathrm{~Hz}$. Cross-correlation of motoneuron activity on both sides gave a phase value of 0.5 for swimming and 1 for synchrony. Both types of measurements matched the exper- 

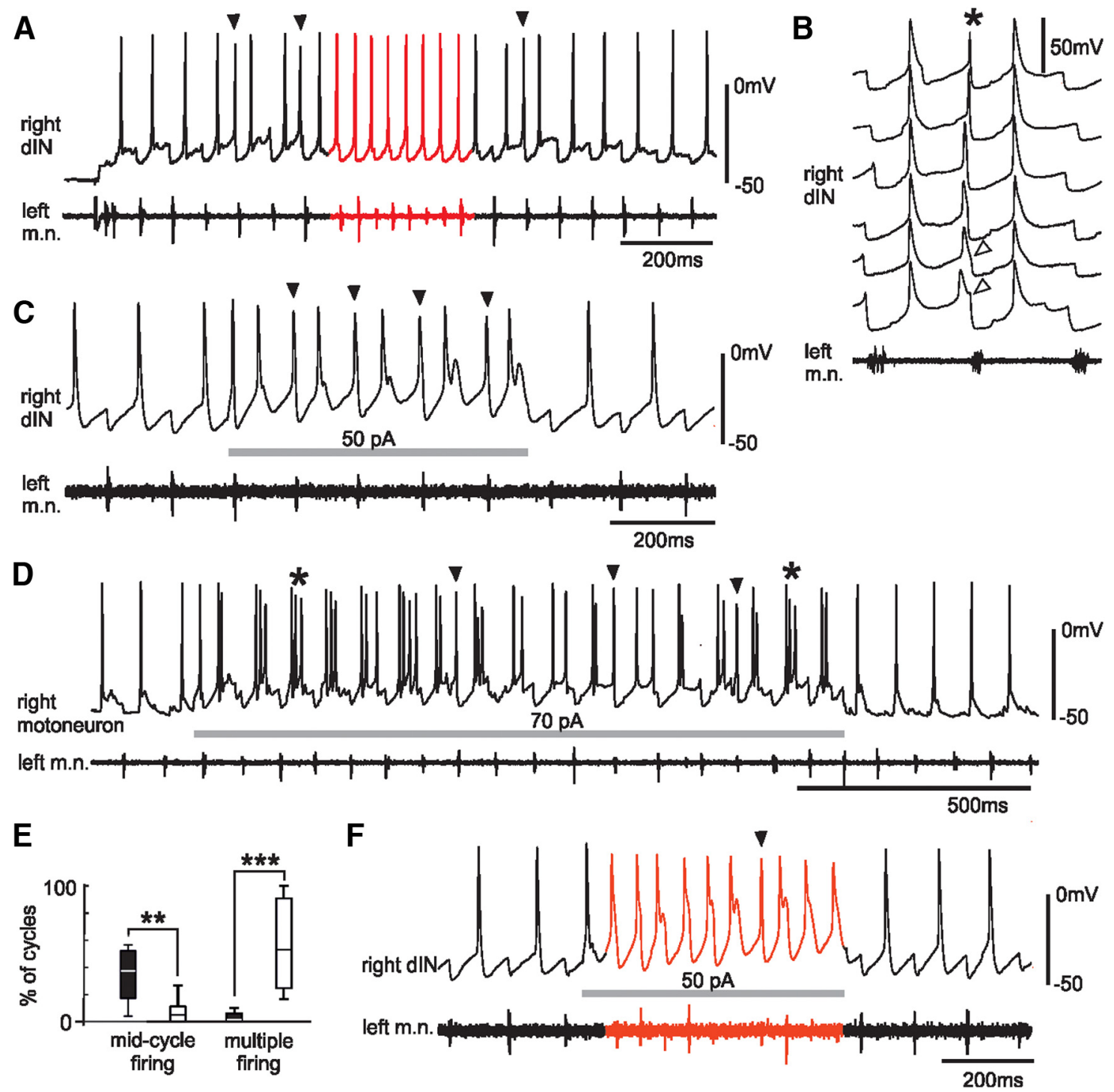

Figure 6. Extra midcycle dIN firing may initiate synchrony. A, Midcycle dIN firing (filled triangles) before and after a synchrony bout. B, Some examples of midcycle dIN firing showing the relative timing of the firing and $\mathrm{CIN}$ inhibition (triangles). Note some spikes are narrowed by inhibition $\left(^{*}\right)$. Lack of midcycle burst in the overlapped left m.n. recording implies the absence of synchrony in the circuit. The illustrated cycles are normalized based on dIN spiking. C, Depolarizing DC injection (gray bar) into a dIN during swimming results in reliable midcycle firing (filled triangles). Note the midcycle spikes do not divide the swimming cycle equally. $D, D C$ injections into a motoneuron result in multiple firing ${ }^{*}$ ) in many cycles and some midcycle firing (filled triangles). $\boldsymbol{E}$, Summary of $12 \mathrm{dINs}$ ' (filled bar) and 16 non-dINs' (unfilled) firing after $D C$ injections during swimming. Significance values as follows: ${ }^{* *} p<0.01$; ${ }^{* * *} p<0.001$. $\boldsymbol{F}$, Midcycle firing of a right-side dIN coincides with synchrony in the left m.n. recording during the DC injection period (filled triangle marks one "swimming-like" cycle; compare with filled triangles in $\boldsymbol{C}$. ( and $\boldsymbol{F}$ are from the same dIN.

imental data. During synchrony, neuronal firing reliability was lower than periods of swimming (paired $t$ test, $p<0.001$ in each case; $n=12$ synchrony bouts; 193 synchrony cycles and equivalent swimming periods compared; Fig. $8 B$ ). AMPA receptor conductance was smaller during synchrony $(4.39 \pm 0.71 \mathrm{nS})$ than during swimming $(5.12 \pm 0.79 \mathrm{nS} ; p<0.01$, paired $t$ test; $n=10 \mathrm{dINs})$. Similarly, combined inhibitory conductance during synchrony $(3.87 \pm 0.86$ $\mathrm{nS}$ ) was smaller than cIN conductance during swimming (5.28 \pm $1.17 \mathrm{nS} ; p<0.01$, paired $t$ test; $n=10 \mathrm{dINs}$; Fig. $8 C$ ). Reducing inhibition strength by up to $60 \%$ failed to convert normal swimming to synchrony (12 trials). Further depression of inhibition made it difficult (three successes in 12 trials at $70 \%$ ) or impossible (above $70 \%$ reduction) to start swimming, similar to what was seen experimentally (Moult et al., 2013).

As shown above, the delay between EPSCs and IPSCs is much shorter during synchrony, and from this we hypothesize that the short delay (Fig. $5 C$ ) reduces neuronal firing reliability and is responsible for the unstable nature of synchrony. To test this, we first advanced dIN activity on one side to further shorten the gap. We injected some brief depolarizing currents into all dINs ( $3 \mathrm{~ms}$, $100 \mathrm{pA}$ ) on the left side to advance neuronal activity by $2.27 \pm$ $0.15 \mathrm{~ms}$ during the fifth synchrony cycle ( 12 trials). This reliably shortened synchrony bouts (related-samples Wilcoxon signed rank test, $p<0.01$; Fig. $9 A$ ) with 10 of 12 bouts ending in the following cycle or with one cycle delay. We next artificially lengthened the EPSC-IPSC gap by increasing the synaptic/conduction delay at dIN to cIN synapses to see whether we could improve synchrony stability. Synaptic delay is about $1 \mathrm{~ms}$ in the tadpole swimming circuit, and dIN spike conduction velocity is $0.36 \mathrm{~mm} / \mathrm{ms}(360 \mu \mathrm{m}$ will increase conduction delay by $1 \mathrm{~ms}$; Soffe et al., 2009). Increasing dIN-cIN transmission/conduction delay is comparable with adding relay interneurons between $\mathrm{dINs}$ 
A

control

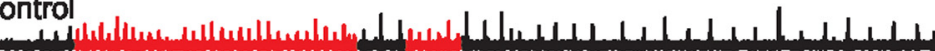

0.5

$0.5 \mu \mathrm{M}$ strychnine

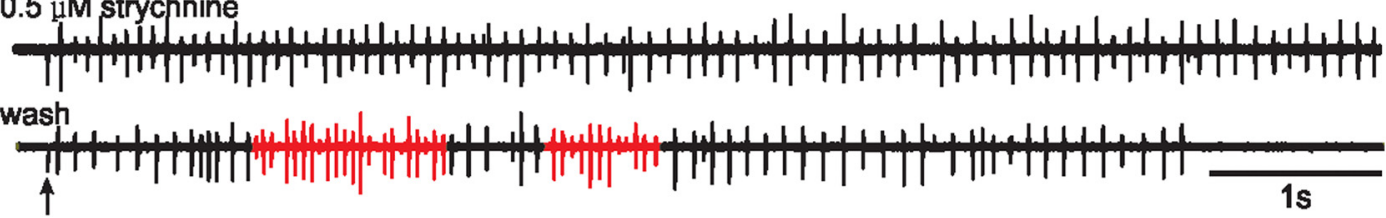

B

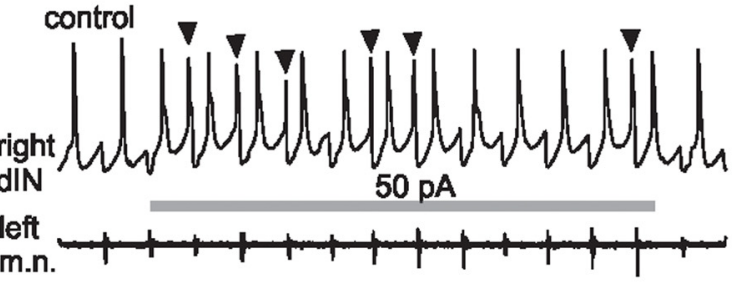

$0.5 \mu \mathrm{M}$ strychnine

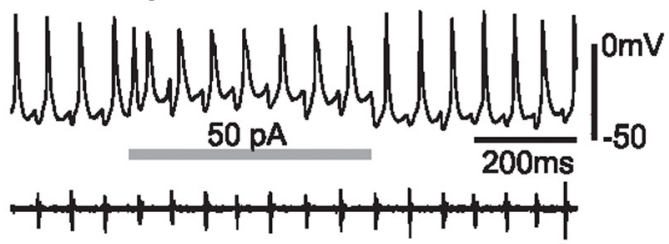

Figure 7. The effects of weakening inhibition on synchrony and dIN firing. $A$, Synchrony bouts in control, when $0.5 \mu \mathrm{m}$ strychnine was applied and during wash. The arrow indicated time of skin stimulation. B, Midcycle dIN firing (arrowheads) induced by DC injection (gray bar) during swimming (control) and its suppression by $0.5 \mu \mathrm{m}$ strychnine.
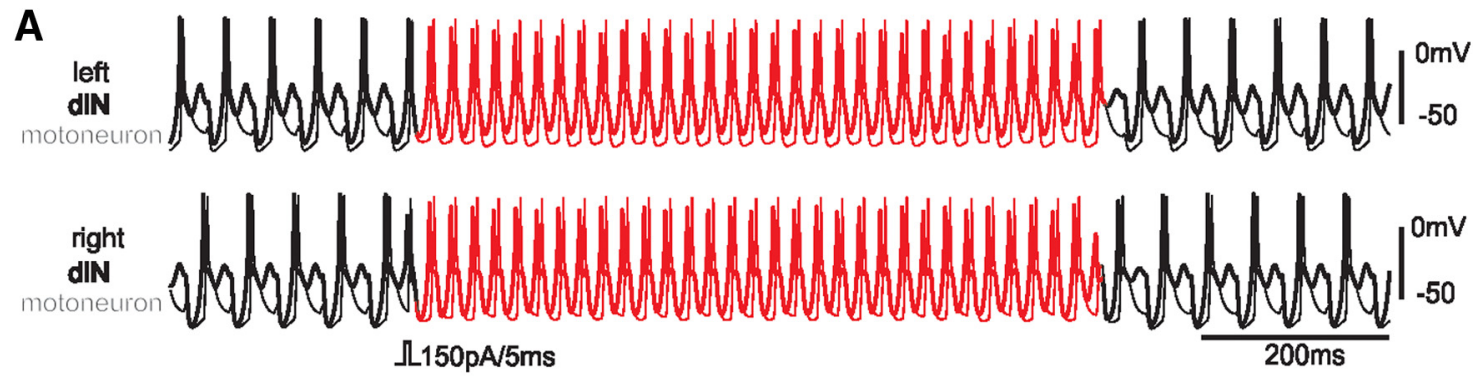

B

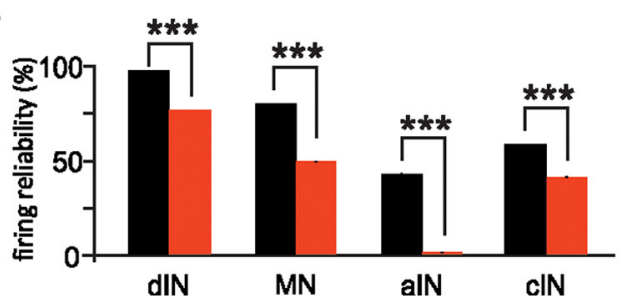

C

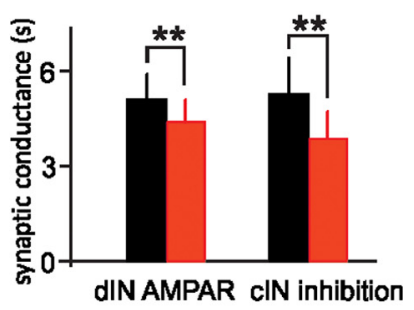

Figure 8. Synchrony initiation and features in a computer model. A, Swimming is converted to self-sustaining synchrony by injecting brief step currents ( $150 \mathrm{pA}, 5 \mathrm{~ms})$ into all dINs on the right once (step). Only one dIN (thick line) and one motoneuron (hairline) traces are shown for simplicity. B, Comparing (PG neuron firing reliabilities during swimming (black) and synchrony (red). $\boldsymbol{C}$, Comparing synaptic conductance in dINs during swimming (black) and synchrony (red). dIN AMPAR conductance is comparable with on-cycle EPSCs during real swimming. cIN inhibition compares midcycle glycinergic conductance during swimming with the combined inhibitory conductance during synchrony (compare Fig. $5, A$ and $B$ ). ${ }^{* * *} p<0.001 ;{ }^{* *} p<0.01$. More details on modeling are in the Results.

and cINs or increasing dIN projection distance (e.g., as is the case when the spinal cord size increases during development). The percentage of simulations with stable synchrony (continuous synchrony from initiation to the end of the $3 \mathrm{~s}$ simulation, $>100$ cycles) initiated by midcycle dIN current injections increased with longer delays (Fig. 9B,C). When the delay was $2 \mathrm{~ms}$ and above, all evoked synchrony bouts were stable. With longer dINcIN synaptic/conduction delays and stable synchrony, all CPG neurons fired more reliably during synchrony $(p<0.001$ in each neuron type, one-way ANOVA; Fig. 9D). We also examined whether there was significant drift of phase during the stable synchrony bouts in simulation with a $2 \mathrm{~ms} \mathrm{dIN}$-cIN delay. Linear regression revealed some small drift in 6 of the 12 simulations (drifting rate, $0.000074 \pm 0.000009$ per cycle; $p<0.05$ ). The modeling, therefore, provides theoretical support that tadpole swimming circuit can generate synchrony and dIN-cIN synaptic/conduction delay can directly determine synchrony stability.

\section{Discussion}

We propose that left-right motor synchrony in tadpoles is mediated by the swimming CPG. The extra midcycle firing of dINs during swimming can initiate synchrony, and the mismatches of timing in left and right activity can convert synchrony to swimming (Fig. 10A). dIN midcycle firing will drive ipsilateral cINs to fire action potentials and produce extra crossed inhibition on the opposite side. This extra inhibition means dINs on both sides will fire simultaneously on postinhibitory rebound and start synchrony. When the activity on one side comes earlier than the opposite side, the crossed inhibition it produces will arrive earlier, inhibiting the activity on the opposite side. 

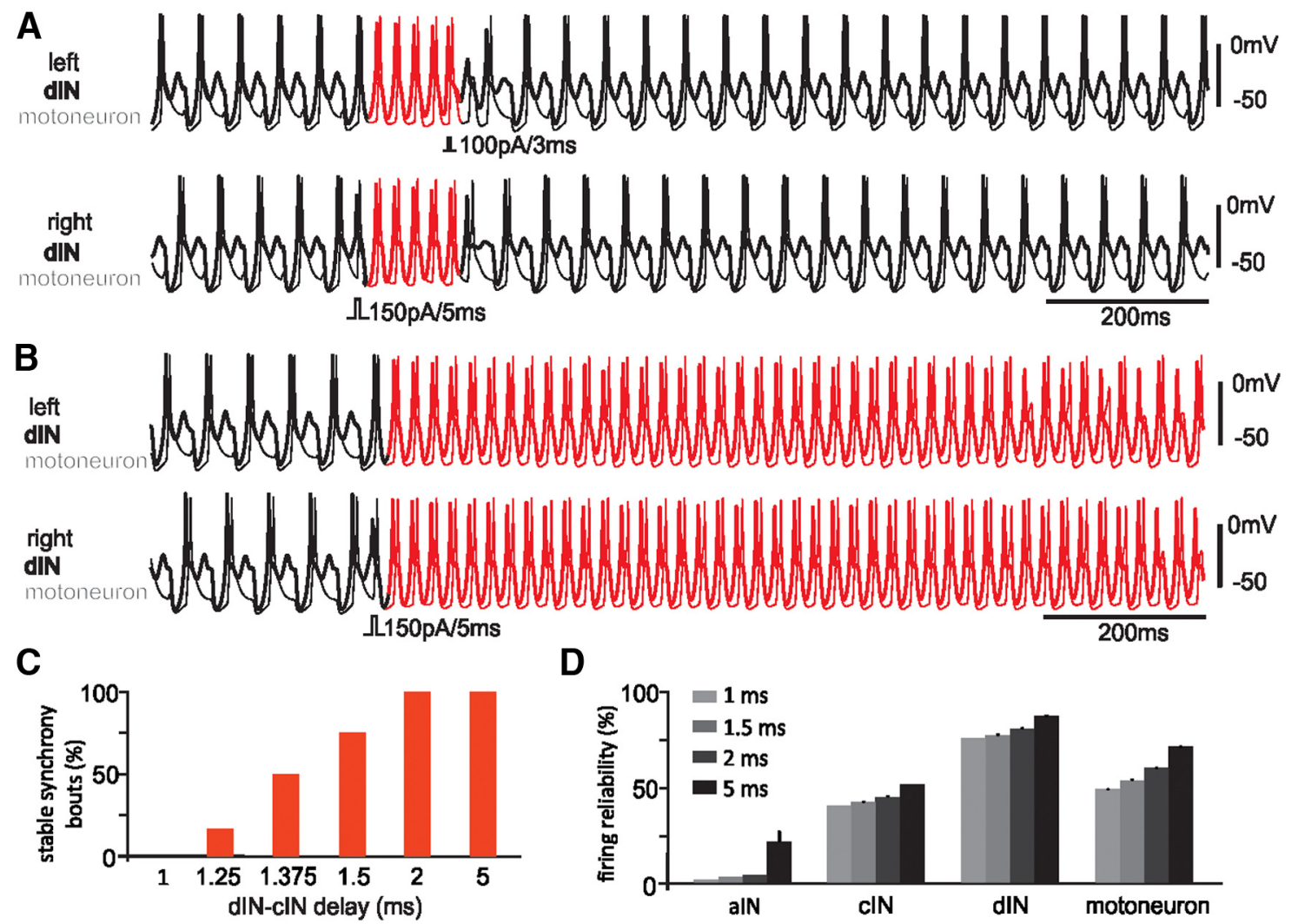

Figure 9. Testing synchrony stability in modeling. $\boldsymbol{A}$, Synchrony evoked by step current injections into dINs on the right is stopped and converted back to swimming by injecting brief step currents once into all dINs on the left (step), which advances neuronal activity by $\sim 2.27 \mathrm{~ms}$. $\boldsymbol{B}$, Increasing $\mathrm{dIN}-\mathrm{cIN}$ synaptic/conduction delay from 1 to $2 \mathrm{~ms}$ converts synchrony to a stable output ( $>100$ cycles, continuous at the end of the $3 \mathrm{~s}$ simulation). C, Percentage of trials with stable, evoked synchrony as in $\boldsymbol{B}$ increases with longer dIN-cIN transmission/conduction delays. $\boldsymbol{D}$, (PG firing reliabilities during synchrony increase with dIN- $\mathrm{CIN}$ delays $(p<0.001$ in each neuron type, one-way ANOVA).

Then, the synchrony pattern breaks down and swimming resumes.

The production of different rhythms in CPGs has been associated with circuit reconfiguration after different sensory inputs (Marder et al., 2005; Briggman and Kristan, 2008; Berkowitz et al., 2010) or under neuromodulatory influences (Rauscent et al., 2009; Combes et al., 2012). The synchrony shown here does not seem to involve reconfiguration of the swimming CPG. Both swimming and synchrony can occur after the same brief skin stimulation, and they involve the same types of neuron, without recruitment of extra or inhibition of existing types of neuron. The same rebound mechanism that sustains swimming appears to operate in synchrony, since dIN firing precedes that of other neurons in both patterns and the rebound times during both outputs are not different. The phase of cIN inhibition during swimming is 0.54 , indicating dIN rebound firing can take place within less than half a cycle. This leaves scope for halving the activity cycle period and may explain the strict relationship between synchrony and swimming frequencies (Fig. 10B). In comparison, the generation of struggling rhythms requires sustained sensory inputs and the recruitment of specialized interneurons, and, furthermore, struggling frequency is not locked to swimming rhythms in any fixed manner (Li et al., 2007).

We have identified midcycle dIN firing as one potential trigger for swimming to switch to synchrony. This extra firing can result from enhanced excitation, e.g., at the beginning of a swimming episode when sensory inputs are still present. In accordance with this, most synchrony bouts occur within $0.5-1 \mathrm{~s}$ of single skin stimulation. Although we have recently shown that pacemaker firing is not sufficient to sustain swimming rhythms, higher excitation may support firing at high frequencies in some dINs (Fig. $6 A-C)$. Once synchrony is initiated, the narrow window for neuronal firing (Fig. $5 C$ ) determines that the firing of some dINs may be prevented by IPSPs (Table 1; Figs. 3D, 5D) and account for the unreliable firing of CPG neurons during synchrony. It also means that synchrony will stop when the activity on both sides drift out of synchrony slightly and explains why most spontaneous synchrony bouts are short. Indeed, drifts in phase with time have been seen only in some longer synchrony bouts. In modeling, evoking midcycle dIN firing during swimming can initiate synchrony, and advancing activity on one side even for only a couple of milliseconds can convert synchrony back to swimming. Synchrony evoked in the model resembles recorded synchrony in frequency and phase features. Artificially broadening the EPSCIPSC delay by increasing dIN-to-cIN synaptic/conduction delay increased CPG firing reliability during synchrony and made synchrony more stable. Therefore, our biological data and modeling both support that the transitions between swimming and synchrony are unlikely to involve circuit reconfiguration.

It was originally proposed that a circuit with two half-centers coupled by reciprocal inhibition could theoretically generate both swimming and synchrony found in the tadpole (Kahn and Roberts, 1982). Later computer modeling, using pairs of neurons with feedback excitation and reciprocal inhibition, suggested that stable synchrony could be evoked by simultaneous sensory activation on both sides or when strong feedback inhibition was added (Roberts and Tunstall, 1990). The previously proposed basic circuit structure has recently been confirmed within the 
A
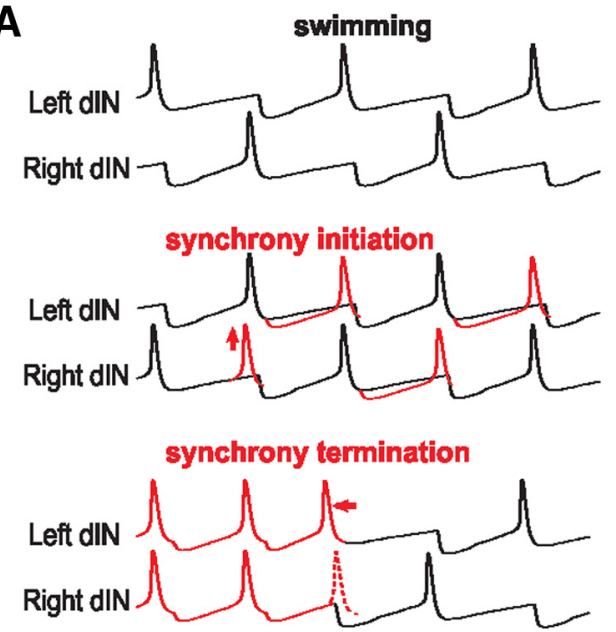

B
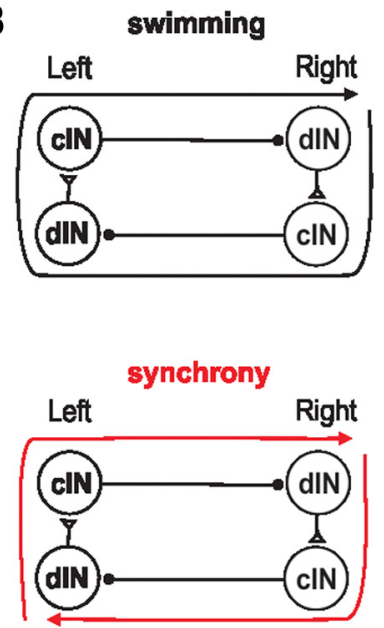

Figure 10. Hypothesis on synchrony initiation and termination. $\boldsymbol{A}$, Illustrative traces show alternating dIN rebound firing in swimming (top traces); simultaneous rebound firing during synchrony triggered by a midcycle spike in the right dIN (red arrow; the other 3 red spikes are "extra" rebound firing; middle traces; and the termination of synchrony when left dIN spiking is advanced (red arrow), the subsequent CIN IPSP arrives earlier and inhibits dIN spiking on the right side (dotted trace; bottom). $\boldsymbol{B}$, Simplified tadpole swimming CPG with alNs and motoneurons omitted to explain neuronal activity within a swimming and synchrony cycle. The triangle synapse is excitatory, and the filled circle is inhibitory. The black arrowed-line indicates activity sequence during swimming. Lines with red arrows indicate activity starts simultaneously in dINs and cycle time is halved during synchrony.

tadpole swimming circuit in a large number of paired whole-cell recordings (Roberts et al., 2010; Li, 2011). This has enabled detailed analyses of neuronal activity and synaptic events during swimming and synchrony here and also allows us to build a computational swimming model of large populations of neurons with biologically realistic properties (Sautois et al., 2007; Borisyuk et al., 2011, 2014). Both our biological and modeling data now provide strong support that reciprocal inhibition, mediated by fast glycinergic synapses, can synchronize network activity. The spontaneous synchrony does not involve different sensory inputs, circuit configuration (Roberts and Tunstall, 1990), or changes in cellular properties like decreased fast potassium currents (Wall and Dale, 1994; Dale, 1995).

The left-right coordination of spinal activity is mediated by crossed inhibition (Kiehn, 2006), but direct commissural excitatory interneurons have been found in some vertebrates. Direct commissural glutamatergic synapses onto motoneurons were reported in mice (Quinlan and Kiehn, 2007). In lamprey, commissural excitatory interneurons have been identified in paired recordings (Buchanan, 1982; Parker and Grillner, 2000), but their role in swimming is primarily unknown (Parker, 2006). Although some interneurons with commissural axons were found to be positive for vesicular glutamate transporters in the larval zebrafish spinal cord (Higashijima et al., 2004), no leftright motor synchrony has been reported in zebrafish. In the tadpole swimming circuit, there is little evidence supporting the existence of crossed excitation from paired recordings except from the sensory interneurons, which are not active during swimming (Dale, 1985; Li et al., 2007). Recording from neurons caudal to hemisections only revealed cIN inhibition during swimming (Soffe and Roberts, 1982).

Blocking glycinergic inhibition may transform alternating flexor-extensor and left-right motor rhythms into bilateral and also flexor-extensor synchrony at lowered frequencies in rodents (Cowley and Schmidt, 1995; Cazalets et al., 1998; Beato and Nistri, 1999). Similar left-right synchrony after inhibition blockade was seen in lamprey (Cohen and Harris-Warrick, 1984; Alford

and Williams, 1989; Hagevik and McClellan, 1994) and mudpuppy (Jovanoviæ et al., 1999). It is thought that in the absence of strong reciprocal inhibition, the weak crossed excitation becomes dominant and couples the activity on the two sides in synchrony (Cohen and Harris-Warrick, 1984; Quinlan and Kiehn, 2007). It is not known whether strychnine could recruit neurons in the circuit that are inhibited in normal rhythms in these cases. The spontaneous synchrony we have studied here cannot be explained by increased weak crossed excitation since there is no evidence for its presence during swimming or synchrony. Instead, inhibition seems to play a role in sustaining spontaneous synchrony as demonstrated by our experiments on the tadpoles with a high tendency for synchronous activity.

Left-right synchrony coupled by crossed excitation has also been reported in some mutant mice. One example comes from mice lacking V2a interneurons (ChX10 DTA), which form ipsilateral excitatory synapses onto commissural interneurons. These mice show galloping gait at high-locomotion speed instead of normal trotting (Crone et al., 2009). This was thought to result from weakened activation of crossed inhibition pathways (Crone et al., 2008, 2009). A second example comes from mutants lacking Ephrin type-A receptor 4, in which ipsilateral excitatory interneurons grow aberrant commissural axons. It was proposed that this led to reciprocal overexcitation of CPG that overcame the coordination mediated by reciprocal inhibition and gave mutants the rabbit-like hopping behavior (Kullander et al., 2001, 2003). Another example involves the deletion of spinal V0 interneurons, which constitute pure commissural interneurons with ascending axons ( $70 \%$ inhibitory, $30 \%$ excitatory). This manipulation could increase left-right synchrony during fictive locomotion (Lanuza et al., 2004). In notochordless Xenopus tadpoles, normal alternation of activity between the left and right side is replaced with synchrony (Clarke et al., 1991). In these tadpoles, many motoneurons have dendrites on both sides of the spinal cord. It is likely that they are excited by dINs on both sides and mediate synchrony.

It is worth pointing out that the aforementioned left-right synchronies were all observed in experimental conditions. Unlike the cases of flying, galloping, hopping, and frog-style swimming, left-right synchrony in axial swimming animals like tadpoles and lamprey is not locomotive. In our modeling, a small increase in dIN-to-cIN synaptic/conduction delay could transform synchrony to a stable regime. Such an increase can take place in the much bigger spinal cord in adult Xenopus. This may provide the mechanism for frog-style swimming, which requires left-right synchrony.

Whereas biological evidence from the spinal cord studies above supports that synchronous activity may be coupled by mutual excitation, some computer modeling has suggested that mutual inhibition can also synchronize network activities. Some studies propose that synchrony takes place when the reciprocal inhibition has slow kinetics (Wang and Rinzel, 1992, 1993; Van Vreeswijk et al., 1994; White et al., 1998). This has gained some biological support from studies of pairs of stomatogastric neu- 
rons artificially connected with reciprocal inhibition using the dynamic-clamp technology (Sharp et al., 1993; Marder, 1998; Elson et al., 2002). A recent modeling study has suggested that fast reciprocal inhibition could also synchronize bursting neurons (Jalil et al., 2010). Similar inhibitory synchronization has also been reported in larger modeled networks. In reticular thalamic (Destexhe et al., 1994), hippocampal, and neocortex (Whittington et al., 1995; Jefferys et al., 1996; Wang and Buzsaki, 1996) computational networks, GABAergic inhibition could synchronize neuronal activity. Biological support for this type of inhibitory synchronization, however, is scarce (Whittington et al., 1995).

Antiphase oscillations and synchrony are arguably the two most common neural rhythms in the CNS. The former is important in locomotion, chewing, and respiration (Kiehn, 2006; Doi and Ramirez, 2008). The latter is common in many cognitive and perceptual functions and shows decrease or enhancement in some neurological or psychiatric disorders (Schnitzler and Gross, 2005; Uhlhaas and Singer, 2006; Brown, 2007; Colgin, 2011; Jiruska et al., 2013). We show that both rhythms are intrinsic to a rebound-based neural circuit that relies on reciprocal inhibition. Additional work needs to thoroughly identify how different factors, including sensory and modulatory inputs and synaptic and neuronal intrinsic properties, stabilize one output preferentially over the other.

\section{References}

Alford S, Williams TL (1989) Endogenous activation of glycine and NMDA receptors in lamprey spinal cord during fictive locomotion. J Neurosci 9:2792-2800. Medline

Beato M, Nistri A (1999) Interaction between disinhibited bursting and fictive locomotor patterns in the rat isolated spinal cord. J Neurophysiol 82:2029-2038. Medline

Berkowitz A (2007) Spinal interneurons that are selectively activated during fictive flexion reflex. J Neurosci 27:4634-4641. CrossRef Medline

Berkowitz A (2008) Physiology and morphology of shared and specialized spinal interneurons for locomotion and scratching. J Neurophysiol 99: 2887-2901. CrossRef Medline

Berkowitz A, Roberts A, Soffe SR (2010) Roles for multifunctional and specialized spinal interneurons during motor pattern generation in tadpoles, zebrafish larvae, and turtles. Front Behav Neurosci 4:36. CrossRef Medline

Borisyuk R, Al Azad AK, Conte D, Roberts A, Soffe SR (2011) Modeling the connectome of a simple spinal cord. Front Neuroinform 5:20. CrossRef Medline

Borisyuk R, Al Azad AK, Conte D, Roberts A, Soffe SR (2014) A developmental approach to predicting neuronal connectivity from small biological datasets: a gradient-based neuron growth model. Plos One 9:e89461. CrossRef Medline

Briggman KL, Kristan WB (2008) Multifunctional pattern-generating circuits. Annu Rev Neurosci 31:271-294. CrossRef Medline

Brown P (2007) Abnormal oscillatory synchronisation in the motor system leads to impaired movement. Curr Opin Neurobiol 17:656-664. CrossRef Medline

Buchanan JT (1982) Identification of interneurons with contralateral, caudal axons in the lamprey spinal cord: synaptic interactions and morphology. J Neurophysiol 47:961-975. Medline

Cazalets JR, Bertrand S, Sqalli-Houssaini Y, Clarac F (1998) GABAergic control of spinal locomotor networks in the neonatal rat. Ann N Y Acad Sci 860:168-180. CrossRef Medline

Clarke JD, Holder N, Soffe SR, Storm-Mathisen J (1991) Neuroanatomical and functional analysis of neural tube formation in notochordless Xenopus embryos; laterality of the ventral spinal cord is lost. Development 112:499-516. Medline

Cohen AH, Harris-Warrick RM (1984) Strychnine eliminates alternating motor output during fictive locomotion in the lamprey. Brain Res 293: 164-167. CrossRef Medline

Colgin LL (2011) Oscillations and hippocampal-prefrontal synchrony. Curr Opin Neurobiol 21:467-474. CrossRef Medline

Combes D, Sillar KT, Simmers J (2012) A switch in aminergic modulation of locomotor CPG output during amphibian metamorphosis. Front Biosci (Schol Ed) 4:1364-1374. Medline

Cowley KC, Schmidt BJ (1995) Effects of inhibitory amino acid antagonists on reciprocal inhibitory interactions during rhythmic motor activity in the in vitro neonatal rat spinal cord. J Neurophysiol 74:1109-1117. Medline

Crone SA, Quinlan KA, Zagoraiou L, Droho S, Restrepo CE, Lundfald L, Endo T, Setlak J, Jessell TM, Kiehn O, Sharma K (2008) Genetic ablation of V2a ipsilateral interneurons disrupts left-right locomotor coordination in mammalian spinal cord. Neuron 60:70-83. CrossRef Medline

Crone SA, Zhong G, Harris-Warrick R, Sharma K (2009) In mice lacking V2a interneurons, gait depends on speed of locomotion. J Neurosci 29: 7098-7109. CrossRef Medline

Dale N (1985) Reciprocal inhibitory interneurones in the Xenopus embryo spinal cord. J Physiol 363:61-70. Medline

Dale N (1995) Experimentally derived model for the locomotor pattern generator in the Xenopus embryo. J Physiol 489:489-510. Medline

Destexhe A, Contreras D, Sejnowski TJ, Steriade M (1994) A model of spindle rhythmicity in the isolated thalamic reticular nucleus. J Neurophysiol 72:803-818. Medline

Dickinson PS, Mecsas C, Marder E (1990) Neuropeptide fusion of two motor-pattern generator circuits. Nature 344:155-158. CrossRef Medline

Doi A, Ramirez JM (2008) Neuromodulation and the orchestration of the respiratory rhythm. Respir Physiol Neurobiol 164:96-104. CrossRef Medline

Elson RC, Selverston AI, Abarbanel HD, Rabinovich MI (2002) Inhibitory synchronization of bursting in biological neurons: dependence on synaptic time constant. J Neurophysiol 88:1166-1176. Medline

Frigon A (2009) Reconfiguration of the spinal interneuronal network during locomotion in vertebrates. J Neurophysiol 101:2201-2203. CrossRef Medline

Getting PA (1989) Emerging principles governing the operation of neural networks. Annu Rev Neurosci 12:185-204. CrossRef Medline

Hagevik A, McClellan AD (1994) Coupling of spinal locomotor networks in larval lamprey revealed by receptor blockers for inhibitory amino acids: neurophysiology and computer modeling. J Neurophysiol 72:1810-1829. Medline

Higashijima S, Schaefer M, Fetcho JR (2004) Neurotransmitter properties of spinal interneurons in embryonic and larval zebrafish. J Comp Neurol 480:19-37. CrossRef Medline

Jalil S, Belykh I, Shilnikov A (2010) Fast reciprocal inhibition can synchronize bursting neurons. Phys Rev E Stat Nonlin Soft Matter Phys 81: 045201. CrossRef Medline

Jefferys JG, Traub RD, Whittington MA (1996) Neuronal networks for induced ' $40 \mathrm{~Hz}$ ' rhythms. Trends Neurosci 19:202-208. CrossRef Medline

Jing J, Weiss KR (2005) Generation of variants of a motor act in a modular and hierarchical motor network. Curr Biol 15:1712-1721. CrossRef Medline

Jiruska P, de Curtis M, Jefferys JG, Schevon CA, Schiff SJ, Schindler K (2013) Synchronization and desynchronization in epilepsy: controversies and hypotheses. J Physiol 591:787-797. CrossRef Medline

Jovanoviæ K, Petrov T, Stein RB (1999) Effects of inhibitory neurotransmitters on the mudpuppy (Necturus maculatus) locomotor pattern in vitro. Exp Brain Res 129:172-184. CrossRef Medline

Kahn JA, Roberts A (1982) Experiments on the central pattern generator for swimming in amphibian embryos. Philos Trans R Soc Lond B Biol Sci 296:229-243. CrossRef Medline

Kiehn O (2006) Locomotor circuits in the mammalian spinal cord. Annu Rev Neurosci 29:279-306. CrossRef Medline

Kimura Y, Okamura Y, Higashijima S (2006) alx, a zebrafish homolog of Chx10, marks ipsilateral descending excitatory interneurons that participate in the regulation of spinal locomotor circuits. J Neurosci 26:56845697. CrossRef Medline

Klein DA, Patino A, Tresch MC (2010) Flexibility of motor pattern generation across stimulation conditions by the neonatal rat spinal cord. J Neurophysiol 103:1580-1590. CrossRef Medline

Kristan WB Jr, Calabrese RL, Friesen WO (2005) Neuronal control of leech behavior. Prog Neurobiol 76:279-327. CrossRef Medline

Kullander K, Croll SD, Zimmer M, Pan L, McClain J, Hughes V, Zabski S, DeChiara TM, Klein R, Yancopoulos GD, Gale NW (2001) Ephrin-B3 is the midline barrier that prevents corticospinal tract axons from recross- 
ing, allowing for unilateral motor control. Genes Dev 15:877-888. CrossRef Medline

Kullander K, Butt SJ, Lebret JM, Lundfald L, Restrepo CE, RydströmA, Klein R, Kiehn O (2003) Role of EphA4 and EphrinB3 in local neuronal circuits that control walking. Science 299:1889-1892. CrossRef Medline

Kupfermann I, Weiss KR (2001) Motor program selection in simple model systems. Curr Opin Neurobiol 11:673-677. CrossRef Medline

Lanuza GM, Gosgnach S, Pierani A, Jessell TM, Goulding M (2004) Genetic identification of spinal interneurons that coordinate left-right locomotor activity necessary for walking movements. Neuron 42:375-386. CrossRef Medline

Liao JC, Fetcho JR (2008) Shared versus specialized glycinergic spinal interneurons in axial motor circuits of larval zebrafish. J Neurosci 28: 12982-12992. CrossRef Medline

Li WC (2011) Generation of locomotion rhythms without inhibition in vertebrates: the search for pacemaker neurons. Integr Comp Biol 51:879889. CrossRef Medline

Li WC, Moult PR (2012) The control of locomotor frequency by excitation and inhibition. J Neurosci 32:6220-6230. CrossRef Medline

Li WC, Soffe SR, Wolf E, Roberts A (2006) Persistent responses to brief stimuli: feedback excitation among brainstem neurons. J Neurosci 26: 4026-4035. CrossRef Medline

Li WC, Sautois B, Roberts A, Soffe SR (2007) Reconfiguration of a vertebrate motor network: specific neuron recruitment and contextdependent synaptic plasticity. J Neurosci 27:12267-12276. CrossRef Medline

Li WC, Roberts A, Soffe SR (2010) Specific brainstem neurons switch each other into pacemaker mode to drive movement by activating NMDA receptors. J Neurosci 30:16609-16620. CrossRef Medline

Marder E (1998) From biophysics to models of network function. Annu Rev Neurosci 21:25-45. CrossRef Medline

Marder E, Bucher D (2007) Understanding circuit dynamics using the stomatogastric nervous system of lobsters and crabs. Annu Rev Physiol 69: 291-316. CrossRef Medline

Marder E, Calabrese RL (1996) Principles of rhythmic motor pattern generation. Physiol Rev 76:687-717. Medline

Marder E, Bucher D, Schulz DJ, Taylor AL (2005) Invertebrate central pattern generation moves along. Curr Biol 15:R685-R699. CrossRef Medline

McLean DL, Fan J, Higashijima S, Hale ME, Fetcho JR (2007) A topographic map of recruitment in spinal cord. Nature 446:71-75. CrossRef Medline

McLean DL, Masino MA, Koh IY, Lindquist WB, Fetcho JR (2008) Continuous shifts in the active set of spinal interneurons during changes in locomotor speed. Nat Neurosci 11:1419-1429. CrossRef Medline

Meyrand P, Simmers J, Moulins M (1991) Construction of a patterngenerating circuit with neurons of different networks. Nature 351:60-63. CrossRef Medline

Meyrand P, Simmers J, Moulins M (1994) Dynamic construction of a neural network from multiple pattern generators in the lobster stomatogastric nervous system. J Neurosci 14:630-644. Medline

Moult PR, Cottrell GA, Li WC (2013) Fast silencing reveals a lost role for reciprocal inhibition in locomotion. Neuron 77:129-140. CrossRef Medline

Nieuwkoop PD, Faber J (1956) Normal tables of Xenopus laevis (Daudin). Amsterdam: North Holland.

Norris BJ, Weaver AL, Morris LG, Wenning A, GarcíaPA, Calabrese RL (2006) A central pattern generator producing alternative outputs: temporal pattern of premotor activity. J Neurophysiol 96:309-326. CrossRef Medline

Parker D (2006) Complexities and uncertainties of neuronal network function. Philos Trans R Soc Lond B Biol Sci 361:81-99. CrossRef Medline

Parker D, Grillner S (2000) The activity-dependent plasticity of segmental and intersegmental synaptic connections in the lamprey spinal cord. Eur J Neurosci 12:2135-2146. CrossRef Medline

Popescu IR, Frost WN (2002) Highly dissimilar behaviors mediated by a multifunctional network in the marine mollusk Tritonia diomedea. J Neurosci 22:1985-1993. Medline

Quinlan KA, Kiehn O (2007) Segmental, synaptic actions of commissural interneurons in the mouse spinal cord. J Neurosci 27:6521-6530. CrossRef Medline

Rauscent A, Einum J, Le Ray D, Simmers J, Combes D (2009) Opposing aminergic modulation of distinct spinal locomotor circuits and their functional coupling during amphibian metamorphosis. J Neurosci 29: 1163-1174. CrossRef Medline

Ritter DA, Bhatt DH, Fetcho JR (2001) In vivo imaging of zebrafish reveals differences in the spinal networks for escape and swimming movements. J Neurosci 21:8956-8965. Medline

Roberts A, Tunstall MJ (1990) Mutual re-excitation with post-inhibitory rebound: a simulation study on the mechanisms for locomotor rhythm generation in the spinal cord of Xenopus embryos. Eur J Neurosci 2:1123. CrossRef Medline

Roberts A, Li WC, Soffe SR (2010) How neurons generate behavior in a hatchling amphibian tadpole: an outline. Front Behav Neurosci 4:16. CrossRef Medline

Roberts A, Conte D, Hull M, Merrison-Hort R, al Azad AK, Buhl E, Borisyuk $\mathrm{R}$, Soffe SR (2014) Can simple rules control development of a pioneer vertebrate neuronal network generating behavior? J Neurosci 34:608 621. CrossRef Medline

Satou C, Kimura Y, Kohashi T, Horikawa K, Takeda H, Oda Y, Higashijima S (2009) Functional role of a specialized class of spinal commissural inhibitory neurons during fast escapes in zebrafish. J Neurosci 29:6780-6793. CrossRef Medline

Sautois B, Soffe SR, Li WC, Roberts A (2007) Role of type-specific neuron properties in a spinal cord motor network. J Comput Neurosci 23:59-77. CrossRef Medline

Schnitzler A, Gross J (2005) Normal and pathological oscillatory communication in the brain. Nat Rev Neurosci 6:285-296. CrossRef Medline

Sharp AA, O'Neil MB, Abbott LF, Marder E (1993) Dynamic clamp: computer-generated conductances in real neurons. J Neurophysiol 69: 992-995. Medline

Soffe SR (1993) Two distinct rhythmic motor patterns are driven by common premotor and motor neurons in a simple vertebrate spinal cord. J Neurosci 13:4456-4469. Medline

Soffe SR, Roberts A (1982) Tonic and phasic synaptic inputs to spinal cord motoneurones active during fictive locomotion in frog embryos. J Neurophysiol 48:1279-1288. Medline

Soffe SR, Roberts A, Li WC (2009) Defining the excitatory neurons that drive the locomotor rhythm in a simple vertebrate: insights into the origin of reticulospinal control. J Physiol 587:4829-4844. CrossRef Medline

Uhlhaas PJ, Singer W (2006) Neural synchrony in brain disorders: relevance for cognitive dysfunctions and pathophysiology. Neuron 52:155-168. CrossRef Medline

Van Vreeswijk C, Abbott LF, Ermentrout GB (1994) When inhibition not excitation synchronizes neural firing. J Comput Neurosci 1:313-321. CrossRef Medline

Wall MJ, Dale N (1994) A role for potassium currents in the generation of the swimming motor pattern of Xenopus embryos. J Neurophysiol 72: 337-348. Medline

Wang XJ, BuzsákiG (1996) Gamma oscillation by synaptic inhibition in a hippocampal interneuronal network model. J Neurosci 16:6402-6413. Medline

Wang X-J, Rinzel J (1992) Alternating and synchronous rhythms in reciprocally inhibitory model neurons. Neural Comput 4:84-97. CrossRef

Wang XJ, Rinzel J (1993) Spindle rhythmicity in the reticularis thalami nucleus: synchronization among mutually inhibitory neurons. Neuroscience 53:899-904. CrossRef Medline

Weimann JM, Marder E (1994) Switching neurons are integral members of multiple oscillatory networks. Curr Biol 4:896-902. CrossRef Medline

Weimann JM, Meyrand P, Marder E (1991) Neurons that form multiple pattern generators: identification and multiple activity patterns of gastric/ pyloric neurons in the crab stomatogastric system. J Neurophysiol 65: 111-122. Medline

White JA, Chow CC, Ritt J, Soto-TreviñoC, Kopell N (1998) Synchronization and oscillatory dynamics in heterogeneous, mutually inhibited neurons. J Comput Neurosci 5:5-16. CrossRef Medline

White RS, Nusbaum MP (2011) The same core rhythm generator underlies different rhythmic motor patterns. J Neurosci 31:11484-11494. CrossRef Medline

Whittington MA, Traub RD, Jefferys JG (1995) Synchronized oscillations in interneuron networks driven by metabotropic glutamate receptor activation. Nature 373:612-615. CrossRef Medline

Wyart C, Del Bene F, Warp E, Scott EK, Trauner D, Baier H, Isacoff EY (2009) Optogenetic dissection of a behavioural module in the vertebrate spinal cord. Nature 461:407-410. CrossRef Medline 